\title{
PRECISION MEASURES OF THE PRIMORDIAL ABUNDANCE OF DEUTERIUM*
}

\author{
Ryan J. Cooke ${ }^{1,7}$, Max Pettini ${ }^{2,3}$, Regina A. Jorgenson ${ }^{4}$, Michael T. MurPhy $^{5}$, and Charles C. Steidel ${ }^{6}$ \\ ${ }^{1}$ Department of Astronomy and Astrophysics, UCO/Lick Observatory, University of California, Santa Cruz, CA 95064, USA; rcooke@ ucolick.org \\ 2 Institute of Astronomy, Madingley Road, Cambridge, CB3 OHA, UK \\ ${ }^{3}$ Kavli Institute for Cosmology, Madingley Road, Cambridge, CB3 OHA, UK \\ ${ }^{4}$ Institute for Astronomy, University of Hawaii, 2680 Woodlawn Drive, Honolulu, HI 96822, USA \\ ${ }^{5}$ Centre for Astrophysics and Supercomputing, Swinburne University of Technology, Hawthorn, Victoria 3122, Australia \\ ${ }^{6}$ California Institute of Technology, MS 249-17, Pasadena, CA 91125, USA \\ Received 2013 August 14; accepted 2013 November 13; published 2014 January 3
}

\begin{abstract}
We report the discovery of deuterium absorption in the very metal-poor $([\mathrm{Fe} / \mathrm{H}]=-2.88)$ damped Ly $\alpha$ system at $z_{\mathrm{abs}}=3.06726$ toward the QSO SDSS J1358+6522. On the basis of 13 resolved D I absorption lines and the damping wings of the $\mathrm{H}_{\mathrm{I}} \mathrm{Ly} \alpha$ transition, we have obtained a new, precise measure of the primordial abundance of deuterium. Furthermore, to bolster the present statistics of precision D/H measures, we have reanalyzed all of the known deuterium absorption-line systems that satisfy a set of strict criteria. We have adopted a blind analysis strategy (to remove human bias) and developed a software package that is specifically designed for precision $\mathrm{D} / \mathrm{H}$ abundance measurements. For this reanalyzed sample of systems, we obtain a weighted mean of $(\mathrm{D} / \mathrm{H})_{\mathrm{p}}=$ $(2.53 \pm 0.04) \times 10^{-5}$, corresponding to a universal baryon density $100 \Omega_{\mathrm{b}, 0} h^{2}=2.202 \pm 0.046$ for the standard model of big bang nucleosynthesis $(\mathrm{BBN})$. By combining our measure of $(\mathrm{D} / \mathrm{H})_{\mathrm{p}}$ with observations of the cosmic microwave background (CMB), we derive the effective number of light fermion species, $N_{\text {eff }}=3.28 \pm 0.28$. We therefore rule out the existence of an additional (sterile) neutrino (i.e., $N_{\mathrm{eff}}=4.046$ ) at $99.3 \%$ confidence $(2.7 \sigma)$, provided that the values of $N_{\text {eff }}$ and of the baryon-to-photon ratio $\left(\eta_{10}\right)$ did not change between BBN and recombination. We also place a strong bound on the neutrino degeneracy parameter, independent of the ${ }^{4} \mathrm{He}$ primordial mass fraction, $Y_{\mathrm{P}}: \xi_{\mathrm{D}}=+0.05 \pm 0.13$ based only on the $\mathrm{CMB}+(\mathrm{D} / \mathrm{H})_{\mathrm{p}}$ observations. Combining this value of $\xi_{\mathrm{D}}$ with the current best literature measure of $Y_{\mathrm{P}}$, we find a $2 \sigma$ upper bound on the neutrino degeneracy parameter, $|\xi| \leqslant+0.062$.
\end{abstract}

Key words: cosmology: observations - primordial nucleosynthesis - quasars: absorption lines

Online-only material: color figures

\section{INTRODUCTION}

We are currently in an exciting era of high-precision cosmology, with most of the "standard" cosmological model parameters now known to within a few percent. In particular, the recent analysis of the cosmic microwave background (CMB) temperature fluctuations recorded by the Planck satellite has found that the present-day density of baryons, $\Omega_{\mathrm{b}, 0}$, contributes just $(2.205 \pm 0.028) / h^{2}$ percent of the critical density (Planck Collaboration 2013), where $h$ is the Hubble constant in units of $100 \mathrm{~km} \mathrm{~s}^{-1} \mathrm{Mpc}^{-1}$. Planck's determination of $\Omega_{\mathrm{b}, 0} h^{2}$, which is now limited by cosmic variance, is the most precise measure of this fundamental physical quantity for the foreseeable future (when derived from the CMB).

For a long time (e.g., Wagoner et al. 1967), it has been appreciated that a complementary measurement of $\Omega_{\mathrm{b}, 0} h^{2}$ can be deduced from the relative abundances of the light elements that were created during big bang nucleosynthesis (BBN). Aside from protons, the only stable nuclei that were produced in astrophysically accessible abundances $\operatorname{are}^{2} \mathrm{H}$ (a.k.a. deuterium,

\footnotetext{
* Based on observations collected at the European Organisation for Astronomical Research in the Southern Hemisphere, Chile (VLT program IDs: 68.B-0115(A), 70.A-0425(C), 078.A-0185(A), 085.A-0109(A)), and the W. M. Keck Observatory, which is operated as a scientific partnership among the California Institute of Technology, the University of California, and the National Aeronautics and Space Administration. The Observatory was made possible by the generous financial support of the W. M. Keck Foundation. Keck telescope time was partially granted by NOAO, through the Telescope System Instrumentation Program (TSIP). TSIP is funded by NSF.

7 Morrison Fellow.
}

D), ${ }^{3} \mathrm{He},{ }^{4} \mathrm{He}$, and trace amounts of ${ }^{7} \mathrm{Li}$ (see Steigman 2007 for a comprehensive review). In particular, considerable effort has been devoted to measuring the abundance of deuterium $(\mathrm{D} / \mathrm{H})$, the mass fraction of ${ }^{4} \mathrm{He}\left(Y_{\mathrm{P}}\right)$, and the abundance of ${ }^{7} \mathrm{Li}$. Of these, the primordial abundance of deuterium is generally accepted as the best "baryometer," owing to its sensitivity and monotonic relationship to $\Omega_{\mathrm{b}, 0} h^{2}$.

The mass fraction of ${ }^{4} \mathrm{He}$, on the other hand, is relatively insensitive to $\Omega_{\mathrm{b}, 0} h^{2}$, but depends quite strongly on the expansion rate of the early universe (typically parameterized in nonstandard models of BBN as an effective number of neutrino species, $\left.N_{\text {eff }}\right)$. At present, this measurement is primarily limited by systematic uncertainties in converting the $\mathrm{He}$ and $\mathrm{H}$ emission lines of metal-poor $\mathrm{H}$ II regions into an estimate of $Y_{\mathrm{P}}$ (see, e.g., Izotov et al. 2013; Aver et al. 2013). Unlike $Y_{\mathrm{P}}$, the ${ }^{7} \mathrm{Li} / \mathrm{H}$ ratio depends modestly on both $\Omega_{\mathrm{b}, 0} h^{2}$ and $N_{\text {eff }}$. However, the observationally inferred value for the "primordial" level, derived from metal-poor Galactic halo stars (Spite \& Spite 1982; Meléndez et al. 2010), is discrepant by a factor of $\sim 3$ compared with the standard model predictions, becoming even more discrepant at the lowest metallicities (Sbordone et al. 2010).

As pointed out recently by Nollett \& Holder (2011) (see also Cyburt 2004), precise measures of the primordial $\mathrm{D} / \mathrm{H}$ ratio can provide interesting bounds on $N_{\text {eff }}$, when combined with a measure of $\Omega_{\mathrm{b}, 0} h^{2}$ from the CMB. The promise of this method was recently demonstrated by Pettini \& Cooke (2012), who obtained the most precise measure of the $\mathrm{D} / \mathrm{H}$ ratio to date, using a metal-poor $([\mathrm{Fe} / \mathrm{H}]=-2.33)$ damped Ly $\alpha$ system (DLA), seen in absorption against a bright background quasar. 
The prospect of measuring the $\mathrm{D} / \mathrm{H}$ ratio in gas clouds seen in absorption along the line of sight to a high-redshift quasar was first pointed out by Adams (1976). This vision was only realized much later, with the advent of 8-10 m class telescopes equipped with high-resolution echelle spectrographs (Burles \& Tytler 1998a, 1998b). Since these first discoveries, a handful of additional cases have been identified (see Pettini \& Cooke 2012 for the "top-ten" list), including one system that appears to be chemically pristine (Fumagalli et al. 2011). One lingering concern with this prime set of measurements is that the dispersion in the reported $\mathrm{D} / \mathrm{H}$ measures is significantly larger than the quoted errors (first noted by Steigman 2001). Indeed, a simple $\chi^{2}$ test reveals that the errors for all $\mathrm{D} / \mathrm{H}$ measurements would need to be scaled upward by $\sim 33 \%$, if the observed dispersion were due to chance alone. An alternative possibility, if the quoted random errors are in fact realistic, is that the analyses may suffer from small systematic biases that are now important to recognize and correct for.

This concern has prompted us to identify the rare handful of systems that afford precise measurements of the primordial abundance of deuterium together with realistic error estimates. This effort is part of our ongoing survey to study the chemistry of the most metal-poor DLAs, described in more detail in Pettini et al. (2008b) and Cooke et al. (2011).

In this paper, we present the Precision Sample of primordial deuterium abundance measurements, consisting of the current best estimates of $\mathrm{D} / \mathrm{H}$ in QSO absorption line systems. We also report a DLA where a new, precise measurement of the primordial deuterium abundance could be obtained. All of these systems have been reanalyzed in a self-consistent manner, taking into account the dominant sources of systematic uncertainty. In the following section, we provide the details of the observations and data reduction procedures for the newly observed DLA and discuss the selection criteria we have adopted to define the Precision Sample. In Section 3, we describe the analysis of the new system with deuterium absorption and estimate its $\mathrm{D} / \mathrm{H}$ ratio. In Section 4 we derive the value of $(\mathrm{D} / \mathrm{H})_{\mathrm{p}}$ from the Precision Sample, discuss its cosmological implications, and consider current limitations in using $\mathrm{D} / \mathrm{H}$ abundance measurements for cosmology. Section 5 deals with the implications of our results for physics beyond the standard model, considering in particular bounds on the effective number of neutrino families and on the lepton asymmetry. Finally, in Section 6, we summarize the main findings from this work.

\section{OBSERVATIONS AND DATA REDUCTION}

\subsection{The DLA toward J1358+6522}

Most newly discovered DLAs are now identified using the low-resolution spectra provided by the Sloan Digital Sky Survey (SDSS). Recent searches for DLAs have yielded several thousand systems (Prochaska \& Wolfe 2009; Noterdaeme et al. 2012). At the modest resolution $(R \sim 2000)$ and signal-to-noise ratio $(\mathrm{S} / \mathrm{N} \sim 20)$ of the SDSS spectra, the metal absorption lines of the most metal-poor DLAs are unresolved and undetected. Follow-up, high spectral resolution observations are thus required to pin down their chemical abundances (see Cooke et al. 2011).

Penprase et al. (2010) were the first to recognize the very metal-poor DLA at redshift $z_{\mathrm{abs}} \simeq 3.0674$ toward the $z_{\mathrm{em}} \simeq 3.173$ quasar SDSS J1358+6522. On the basis of their intermediate-resolution observations $\left(60 \mathrm{~km} \mathrm{~s}^{-1}\right.$ FWHM) with the Keck Echellette Spectrograph and Imager, these au- thors concluded that this DLA has a metallicity of $[\mathrm{Fe} / \mathrm{H}]$ $\leqslant-3.02$ and is thus among the most metal-poor known (Cooke et al. 2011). We re-observed this QSO with the Keck High Resolution Echelle Spectrometer (HIRES; Vogt et al. 1994) using the red cross-disperser to perform an accurate chemical abundance analysis (Cooke et al. 2012). The HIRES data pinned down the metallicity of the DLA $([\mathrm{Fe} / \mathrm{H}]=-2.84 \pm 0.03)$ and provided the first indication for the presence of a handful of resolved D I absorption lines.

Taking advantage of the high efficiency of the HIRES UV cross-disperser at blue wavelengths, we then performed dedicated HIRES observations with the goal of obtaining a precise measure of the deuterium abundance. Our observations included a total of 24,300 s on 2012 May 11, (divided equally into nine exposures) and an additional 29,750 s on 2013 May 3 and 5. An identical instrument setup was employed for both runs; we used the $\mathrm{C} 1$ decker $\left(7^{\prime \prime} \times 0.861\right.$, well matched to the seeing conditions of $\left.00^{\prime \prime} 6-00^{\prime \prime} 8\right)$, which delivers a nominal spectral resolution of $R \sim 48,000\left(\equiv 6.3 \mathrm{~km} \mathrm{~s}^{-1}\right.$ FWHM) for a uniformly illuminated slit. Our setup covers the wavelength range $3650-6305 \AA$, with small gaps near $4550 \AA$ and $5550 \AA$ due to the gaps between the HIRES detector chips. The exposures were binned on-chip $2 \times 2$.

The data were reduced following the standard procedures of bias subtraction, flat-fielding, order definition, and extraction. The data were mapped onto a vacuum, heliocentric wavelength scale from observations of a ThAr lamp that bracketed the science exposures. To test the quality of the data reduction, we reduced the data using two pipelines: MAKEE, which is maintained by T. Barlow, ${ }^{8}$ and HIREDUX, which is maintained by $\mathrm{J}$. X. Prochaska. ${ }^{9}$ We found that the skyline subtraction performed by HIREDUX was superior to that of MAKEE and substantially improved the $\mathrm{S} / \mathrm{N}$ of the spectra at blue wavelengths. This was very important for the removal of telluric features near the redshifted D I and H I transitions of the DLA. All data analyzed and presented herein for J1358+6522 were reduced with HIREDUX. The individual orders were combined with UVES_POPLER, which is maintained by M. T. Murphy. ${ }^{10}$ As a final step, we fluxcalibrated the echelle data by comparison to the SDSS data. The combined $\mathrm{S} / \mathrm{N}$ of the final spectrum per $2.5 \mathrm{~km} \mathrm{~s}^{-1}$ pixel is $\sim 30$ near $4000 \AA, \sim 45$ at $5000 \AA$, and $\sim 20$ near $6000 \AA$. The reduced, flux-calibrated spectrum is presented in Figure 1, where the red tick marks indicate the wavelengths of the redshifted $\mathrm{H}$ I Lyman series transitions.

\subsection{Literature D/H QSO Absorption Line Systems}

\subsubsection{Selection Criteria}

In this section, we outline the strict set of rules that we have used to define the Precision Sample. Our goal is to identify the small handful of systems currently known where the most accurate and precise measures of $\mathrm{D} / \mathrm{H}$ can potentially be obtained. The set of restrictions we applied are as follows:

1. We require that the $\mathrm{H}_{\mathrm{I}} \mathrm{Ly} \alpha$ absorption line must exhibit Lorentzian damping wings. This criterion is satisfied for absorption-line systems with $\log N(\mathrm{H} \mathrm{I}) / \mathrm{cm}^{-2} \geqslant 19$. Such absorption lines lie on the damping regime of the curve of growth, where $N(\mathrm{HI})$ can be derived independently of the

\footnotetext{
8 MAKEE is available at http://www.astro.caltech.edu/ tb/makee/ 9 HIREDUX can be obtained from http://www.ucolick.org/ xavier/HIRedux/index.html

10 UVES_POPLER can be downloaded from

http://astronomy.swin.edu.au/ mmurphy/UVES_popler/
} 


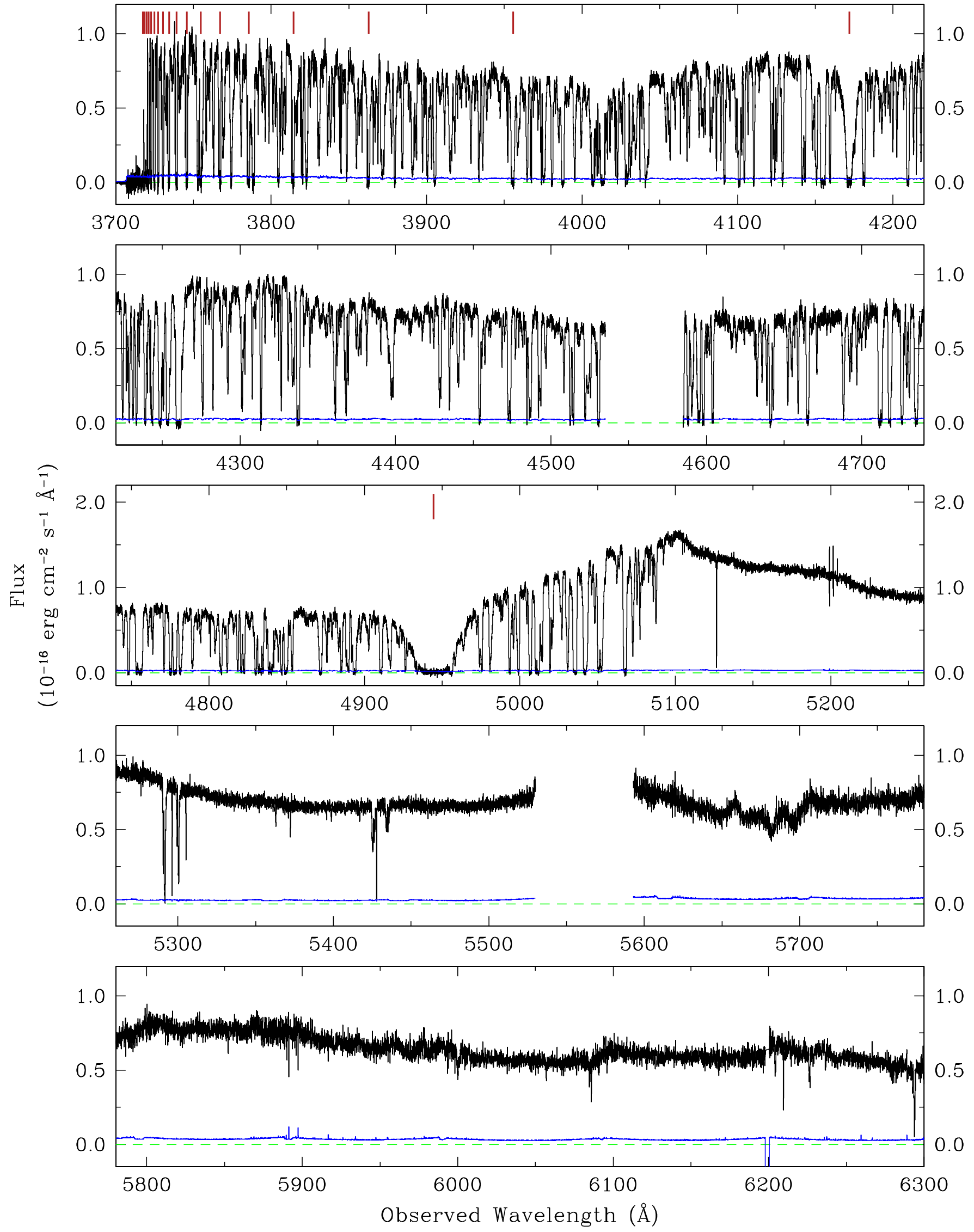

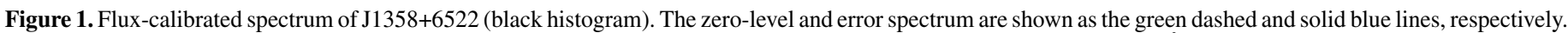

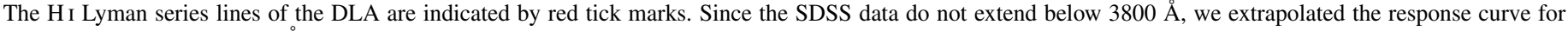
fluxing the data a further $100 \AA$ to the blue.

(A color version of this figure is available in the online journal.) 
cloud model; the damping wings uniquely determine the total H I column density.

2. Due to the power of the H I Ly $\alpha$ transition in establishing the total H I column density, we require that the wings of this transition are not strongly blended with nearby, unrelated, strong $\mathrm{H}$ I absorption systems. If there exist additional strong H I absorption systems nearby, we impose that the edges of their Ly $\alpha$ absorption troughs should be separated by at least $500 \mathrm{~km} \mathrm{~s}^{-1}$, and that all such absorption systems should be modeled simultaneously.

3. At least two, resolved and apparently unblended, optically thin transitions of D I must be available. This ensures that the total D I column density can be measured accurately and independently of the cloud model.

4. The data must have been obtained with a high-resolution echelle spectrograph, with $R \geqslant 30,000$ (i.e., $v_{\text {FWHM }} \leqslant$ $\left.10 \mathrm{~km} \mathrm{~s}^{-1}\right)$, to resolve the broadening of the D I lines, and recorded at $\mathrm{S} / \mathrm{N} \gtrsim 10 \mathrm{pixel}^{-1}\left(\sim 3 \mathrm{~km} \mathrm{~s}^{-1}\right)$ at both Ly $\alpha$ and the weakest D I absorption line used in the analysis.

5. Several unblended metal lines with a range of oscillator strengths for a given species (ideally $\mathrm{O}$ I and Si II) must be present if there is only one optically thin D I transition in order to determine the velocity structure of the absorbing cloud and ensure that the presence of any partly ionized gas (which may contribute to the $\mathrm{H}_{\mathrm{I}}$ column density) is accurately modeled. However, if there exist at least two optically thin DI transitions, metal lines are not strictly required.

Of course, we are also limited by data that we, and others, have access to. Data that are not publicly available on archives could not be used in this study. In total, there are four systems in the literature that meet the above criteria. We now give a brief description of these systems.

$$
\text { 2.2.2. } H S O 105+1619, z_{\mathrm{abs}}=2.53651
$$

Keck+HIRES observations of the QSO HS 0105+1619 were obtained as follows: $1800 \mathrm{~s}$ in 1999 (Program ID: U05H, PI: A. Wolfe), 85,000 s in 1999-2000 by O'Meara et al. (2001), and 21,500 s in 2005 (Program ID: G10H, PI: D. Tytler). The data obtained by O'Meara et al. (2001) are not available from the Keck Observatory Archive. Thus, we have reanalyzed only the last data set, which, in any case, was obtained with a much-improved HIRES detector. As reported by O'Meara et al. (2001), the spectrum shows nine clean H I transitions in the Lyman series together with four optically thin D I absorption lines; the absorption is confined to a single velocity component at $z_{\mathrm{abs}}=2.536509$. The first ions exhibit slightly asymmetric profiles, arising from ionized gas blueshifted by $7.1 \mathrm{~km} \mathrm{~s}^{-1}$ relative to the main component (see Figure 8 in the Appendix). The H I column density of this ionized component contributes just 0.01 dex to the total column density $\log N(\mathrm{HI}) /$ $\mathrm{cm}^{-2}=19.42 \pm 0.01$. The data and best-fitting model are shown in Figure 8.

$$
\text { 2.2.3. } Q 0913+072, z_{\mathrm{abs}}=2.61829
$$

The quasar Q0913+072 intersects a DLA with $\log N(\mathrm{HI}) /$ $\mathrm{cm}^{-2}=20.34 \pm 0.04$ at $z_{\mathrm{abs}}=2.61829$. Q0913+072 was observed with the Ultraviolet and Visual Echelle Spectrograph (UVES) on the European Southern Observatory's (ESO) Very Large Telescope (VLT) facility in 2002 (Program ID: 68.B0115(A), PI: P. Molaro), and then again in 2007 to obtain UV coverage down to the Lyman limit (Program ID: 078.A-0185(A),
PI: M. Pettini), resulting in a total exposure time of 77,500 s. The details of this system's chemical composition were reported by Pettini et al. (2008b) (see also Erni et al. 2006), and the analysis of the D I lines was presented by Pettini et al. (2008a); with $[\mathrm{O} / \mathrm{H}]=-2.40$ this is currently the most metal-poor DLA known with resolved D I lines. There is a small column density of ionized gas at velocities near that of the DLA; we have carefully modeled its contribution to the overall absorption in the metal lines and the Hi Lyman series (see Figure 9 in the Appendix).

Since Q0913+072 does not have an SDSS spectrum from which we could perform a flux calibration, we obtained a set of VLT+FORS2 (Focal Reducer/low dispersion Spectrograph 2) exposures from the VLT archive to flux-calibrate the UVES spectrum (Program ID: 70.A-0425(C), PI: L. Wisotzki). The FORS2 data were reduced following standard procedures, using the software routines from the ESO FORS2 data reduction pipeline. ${ }^{11}$ The UVES data and the corresponding model fits are shown in Figure 9.

$$
\text { 2.2.4. } J 1419+0829, z_{\mathrm{abs}}=3.04973
$$

The chemical properties of this DLA were discussed by Cooke et al. (2011), while Pettini \& Cooke (2012) reported the analysis of the deuterium lines. This system currently holds the record for the most precise determination of the $\mathrm{D} / \mathrm{H}$ ratio. The analysis technique developed in Pettini \& Cooke (2012) is the same as that employed here (see Section 3.2), except for two aspects: (1) in the present work all QSO spectra were analyzed "blind," i.e., without knowledge of the value of $\mathrm{D} / \mathrm{H}$ until the line-fitting procedure was concluded; and (2) we now allow for an arbitrary continuum fitting with Legendre polynomials, rather than the combination of powerlaw continuum and Gaussian emission lines used by Pettini \& Cooke (2012). To maintain consistency with the other DLAs investigated here, we have reanalyzed this system with the new prescription for the continuum definition, and the analysis was performed blind. We found that the final value of $\mathrm{D} / \mathrm{H}$ from this new analysis deviated by just 0.005 dex from that reported by Pettini \& Cooke (2012), with an identical estimate for the error. This small shift is due to our new approach for dealing with the systematic uncertainty in the continuum level.

$$
\text { 2.2.5. J1558-0031, } z_{\mathrm{abs}}=2.70242
$$

The data for J1558-0031 consist of an 11,300 s Keck+HIRES spectrum obtained in 2006 (Program ID: U152Hb, PI: J. X. Prochaska), first reported by O'Meara et al. (2006). With $\log N(\mathrm{HI}) / \mathrm{cm}^{-2}=20.67 \pm 0.05$, this $z_{\mathrm{abs}}=2.70242$ DLA is the highest H I column density system currently known with at least one optically thin D I absorption feature. There exists another metal-poor DLA along this sightline, at $z_{\text {abs }}=2.629756$, that also exhibits damped Ly $\alpha$ absorption $\left(\log N(\mathrm{HI}) / \mathrm{cm}^{-2}=\right.$ $19.726 \pm 0.007)$. This second system contains a single absorption component, as evidenced by several Si II absorption lines (see bottom-right panel of Figure 10 in the Appendix). Although D I absorption is only seen in the higher redshift DLA, we include the $\mathrm{H}$ I and metal lines of both DLAs in the fitting procedure, since the damping wings of the two DLAs overlap slightly. Portions of the HIRES spectrum, together with the best-fitting model, are reproduced in Figure 10. Unlike the O'Meara et al.

\footnotetext{
11 We used version 3.9.6, obtained from http://www.eso.org/sci/software/pipelines/
} 
(2006) analysis, which uses data acquired with both Magellan+MIKE and Keck+HIRES, our analysis exclusively uses the Keck+HIRES data described above.

\section{PROFILE ANALYSIS}

The analysis we have adopted to measure the $\mathrm{D} / \mathrm{H}$ ratio is an improvement over previous studies, with two main differences: (1) the profile fitting and $\chi^{2}$ minimization process was performed with new, purpose-built software routines; and (2) we have tried to identify the dominant systematics and have attempted to account for these effects. Before we discuss the details of our line profile analysis, we briefly describe the important aspects of the new software we have written to measure $\mathrm{D} / \mathrm{H}$ in metal-poor DLAs. We will focus our discussion on the newly discovered system with a precise value of the $\mathrm{D} / \mathrm{H}$ abundance (J1358+6522), but we stress that all five DLAs were analyzed in an identical manner.

\subsection{Line Profile Fitting Software}

To account for the dominant systematics that could potentially affect the measurement of $\mathrm{D} / \mathrm{H}$, we have developed a new suite of software routines written in the PYTHON software environment. Our Absorption LIne Software (ALIS; described in more detail by R. J. Cooke, in preparation) uses a modified version of the MPFIT package (Markwardt 2009). MPFIT employs a Levenberg-Marquardt least-squares minimization algorithm to derive the model parameters that best fit the data (i.e., the parameters that minimize the difference between the data and the model, weighted by the error in the data). Unlike the software packages that have previously been used to measure $\mathrm{D} / \mathrm{H}$, ALIS has the advantage of being able to fit an arbitrary emission profile for the quasar while simultaneously fitting to the absorption from the DLA; any uncertainty in the continuum is therefore automatically folded into the final uncertainty in the $\mathrm{D} / \mathrm{H}$ ratio. For all transitions, we use the atomic data compiled by Morton (2003).

\subsection{Analysis Technique for Measuring $D / H$}

We first highlight one of the main strengths of our analysis that, to our knowledge, has not been implemented in previous $\mathrm{D} / \mathrm{H}$ studies. In order to remove human bias from the results, we have adopted a blind analysis strategy, whereby the only information revealed during the profile-fitting process is the best-fitting $\chi^{2}$ value, and plots of the corresponding model fits to the data; all of the parameter fitting-results are entirely hidden from view. Only after the analysis converged on the best-fitting model were the full results of the model fit uncovered, and these are the final values quoted here. No further changes were made to the model fit nor data reduction after the results were revealed.

To allow for an efficient computation, a small wavelength interval (typically $\pm 300 \mathrm{~km} \mathrm{~s}^{-1}$ ) around each absorption line of interest was extracted. For each of these intervals, we manually identified the regions to be used in the fitting process by selecting pixels that we attribute to either the quasar continuum or the DLA's absorption lines. In some cases, it was also necessary to model unrelated absorption lines in the fitting procedure if there was significant line blending.

We have also attempted to account for the main systematics and limitations that might affect the reported $\mathrm{D} / \mathrm{H}$ value. The dominant systematics likely include the instrumental broadening function, the cloud model, and the placement of the quasar continuum and zero level. We marginalize over the first of these systematics by assuming that the instrumental profile is a Gaussian, ${ }^{12}$ where the Gaussian FWHM was allowed to be a free model parameter during the $\chi^{2}$ minimization process.

Any uncertainty or bias in the choice of the cloud model is overcome by our selection criteria listed in Section 2.2.1. Specifically, DLAs have two advantages over other absorptionline systems for the determination of the $\mathrm{D} / \mathrm{H}$ ratio, both stemming from their high H I column densities: (1) the H I Ly $\alpha$ line exhibits well-defined Lorentzian damping wings ${ }^{13}$ from which the total H I column density can be deduced precisely and independently of the cloud model, and (2) many D I transitions in the Lyman series are normally detectable. With their widely varying oscillator strengths, these multiple D I lines can pin down the D I column density very precisely; the availability of unsaturated, high-order lines ensures that the value of $\mathrm{D} / \mathrm{H}$ thus deduced does not depend on the detailed kinematic structure of the gas (i.e., the cloud model). Neither of these two advantages applies to Lyman limit systems whose H i column density can be three orders of magnitude lower than that of DLAs.

Finally, if the background level is not accurately determined, a small offset can systematically affect the derived $\mathrm{D} / \mathrm{H}$ value. We have overcome this concern by allowing the zero level to be fit during the minimization process (assuming the offset to be the same at all wavelengths).

Arguably, the above systematic effects are sub-dominant compared to the choice of the continuum level. One of the improvements over previous analyses is that we have not specified a priori the level of the quasar continuum. Instead, we have marginalized over the continuum level during the fitting process by fitting Legendre polynomials to the flux-calibrated quasar continuum. The order of the polynomial was chosen to be sufficiently large to allow flexibility in arbitrarily defining the continuum level, while ensuring that the continuum was not being overfit to the noise (examples are shown in the top panels of Figures 2, 8, 9, and 10).

We emphasize that by including the above systematics into the fitting process, the uncertainties in the continuum, zero level, and instrumental broadening are incorporated into the final uncertainty in the derived $\mathrm{D} / \mathrm{H}$ value. To summarize, we have marginalized over the continuum, zero level, and instrumental FWHM, while simultaneously fitting for all of the DLA's unblended metal lines, the DLA's Ly $\alpha$ transition, and the entire $\mathrm{H}_{\mathrm{I}}$ and D I Lyman series. Our analysis therefore includes all of the available information that can help pin down the $\mathrm{D} / \mathrm{H}$ value, and it accounts for the systematics that are currently thought to contribute the most to the overall error budget. We finally point out that every $\mathrm{H}_{\mathrm{I}}$ component used to fit the DLA was forced to have the same $\mathrm{D}$ I/H I ratio. We have therefore fit directly to the $\mathrm{D}$ I/H I value, rather than deriving separately the column densities of D I and H I. All absorption lines were modeled as Voigt profiles where the line broadening has both thermal and turbulent contributions. All of the DLAs in our survey were first modeled with a single absorption component. If the metal lines exhibited an asymmetric profile, an additional component was included to improve the quality of the fit.

To confirm that the model parameters had converged to their best-fitting values, we performed a convergence test; once the

\footnotetext{
12 We confirmed that emission lines from the ThAr wavelength calibration spectrum were well fit by a Gaussian profile.

${ }^{13}$ Lee (2013) recently suggested that the wings of the damped Ly $\alpha$ absorption line exhibit an asymmetric deviation from a Lorenzian. Such a deviation from a Lorentzian profile would be marginalized over during the arbitrary continuum-fitting process described below and is thus expected to have a negligible impact on our results.
} 

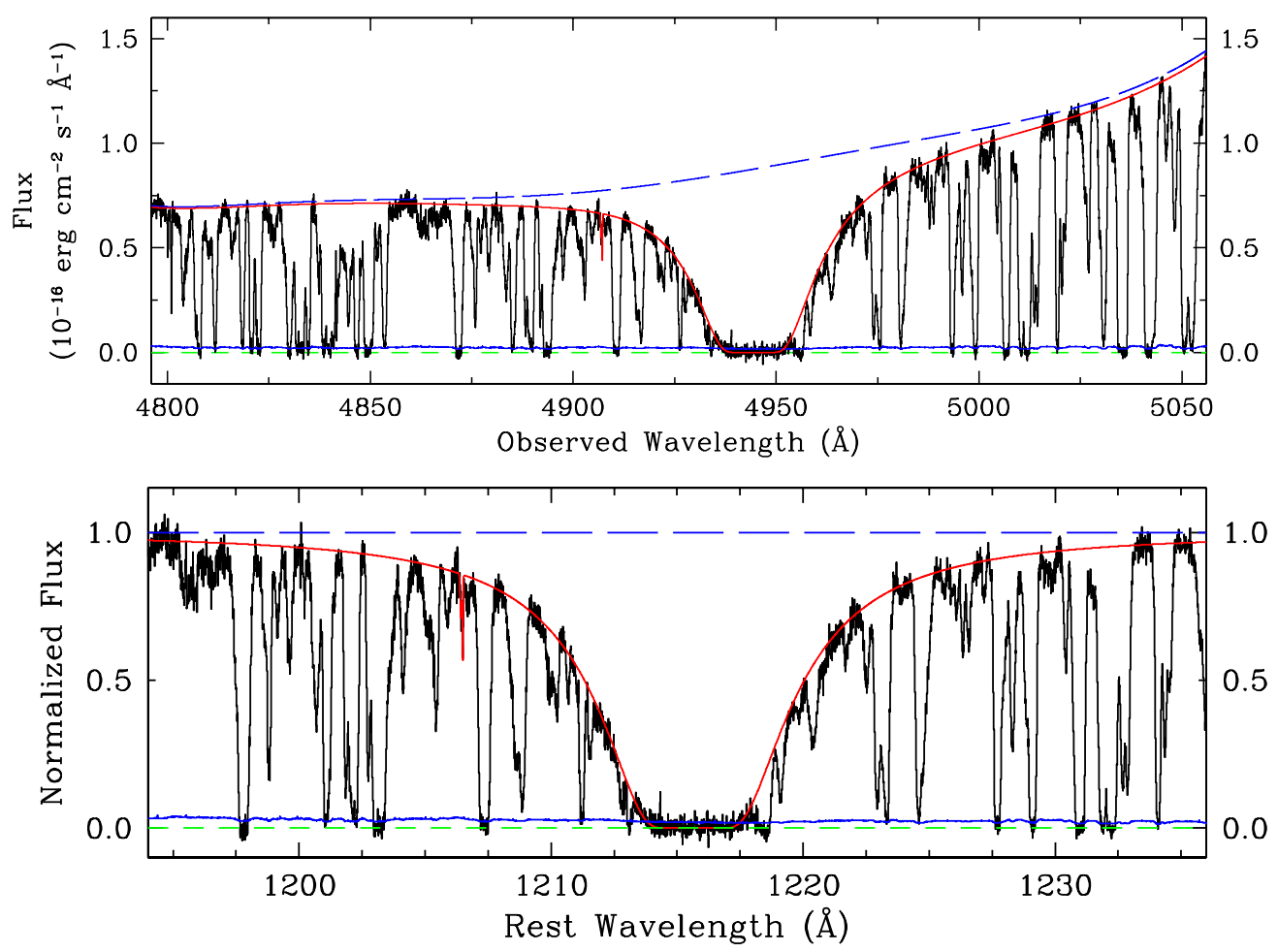

Figure 2. The top panel displays a portion of the flux-calibrated HIRES spectrum near the damped Ly $\alpha$ line at $z_{\text {abs }}=3.06726$ toward J1358+6522 (black histogram) together with the error spectrum (continuous blue line). The dashed green line marks the best-fitting zero level of the data, and the dashed blue line shows the best-fitting continuum level. The solid red line shows the overall best-fitting model to the DLA. The bottom panel shows a zoom-in of the data and model; the weak absorption feature that we have modeled on the blue wing of Ly $\alpha$ is Si III $\lambda 1206.5$ at the redshift of the DLA ( $4907 \AA$ in the observed frame).

(A color version of this figure is available in the online journal.)

difference in the $\chi^{2}$ between successive iterations was $<0.01$, the parameter values were stored and the $\chi^{2}$ minimization recommenced with a tolerance of $10^{-3}$. Once a successive iteration reduced the $\chi^{2}$ by $<10^{-3}$, the minimization was terminated and the parameter values from the two convergence criteria were compared. If all parameter values differed by $<0.2 \sigma$ (i.e., $20 \%$ of the parameter error), then the model fit has converged.

As a final step, we repeated the $\chi^{2}$ minimization process 20 times, perturbing the starting parameters of each run by the covariance matrix. This exercise ensures that our choice of starting parameters does not influence the final result. We found that the choice of starting parameters has a negligible contribution to the error on D I/H I (typically $0.002 \mathrm{dex}$ ), but can introduce a small bias (again, typically $0.002 \mathrm{dex}$ ). We have accounted for this small bias in all of the results quoted herein.

\subsection{Component Structure}

Most of the narrow, low-ionization metal lines of the DLA toward J1358+6522 consist of a single component at $z_{\text {abs }}=3.067259$. A second weaker component, blueshifted by $17.4 \mathrm{~km} \mathrm{~s}^{-1}\left(z_{\mathrm{abs}}=3.06702\right)$, contributes to Si III $\lambda 1206.5$ and to the strongest $\mathrm{C}$ II and Si II lines. Evidently, this weaker absorption arises in nearby ionized gas.

In fitting the absorption lines, we tied the redshift, turbulent Doppler parameter, and kinetic temperature of the gas to be the same for the metal, Di, and Hi absorption lines. We allowed all of the cloud model parameters to vary, while simultaneously fitting for the continuum near every absorption line. Relevant parameters of the best-fitting cloud model so determined are collected in Table 1. Figures 2, 3, and 4 compare the data and model fits for, respectively, the damped $\operatorname{Ly} \alpha$ line, the full Lyman series, and selected metal lines. [Since the metal lines analyzed here are the same as those shown in Figure 1 of Cooke et al. (2012), albeit now with a higher S/N, we only present a small selection of them in Figure 4 to avoid repetition]. The best-fitting chi-squared value for this fit is also provided for completeness. ${ }^{14}$

Returning to Table 1, it can be seen that we found it necessary to separate the main absorption into two separate components, labeled $1 \mathrm{a}$ and $1 \mathrm{~b}$ in the table. A statistically acceptable fit ${ }^{15}$ to the metal, D I and H I lines could not be achieved with a single absorbing cloud in which the turbulent broadening is the same for all species and the thermal broadening is proportional to the square root of the ion mass (i.e., $b_{\mathrm{th}}^{2}=2 K T / \mathrm{m}$, where $K$ is the Boltzmann constant). The main absorption component of this DLA appears to consist of two "clouds" with very similar redshifts, temperatures, and $\mathrm{H}$ I column densities, but with significantly different turbulence parameters (see Table 1). The turbulent broadening for component 1a is bounded by the metal lines, whereas the thermal broadening is bounded by the relatively narrow $\mathrm{H}$ I line profiles. This combination of turbulent and thermal broadening is unable to reproduce the observed widths of the strongest D I lines, which require an additional component with a larger contribution of turbulent broadening. Surprisingly, metal absorption is only seen in the

\footnotetext{
14 We caution that the quoted chi-squared value is likely underestimated in our analysis because: (1) there is some degree of correlation between neighboring pixels that is not accounted for in the error spectrum, and (2) the continuum regions selected tend to have lower fluctuations about the mean than average. 15 The addition of an extra absorption component (i.e., three components as opposed to two, and four additional free parameters) reduces the minimum chi-squared value by $\Delta \chi_{\min }^{2} \simeq 660$, which is highly significant (see, e.g., Lampton et al. 1976).
} 

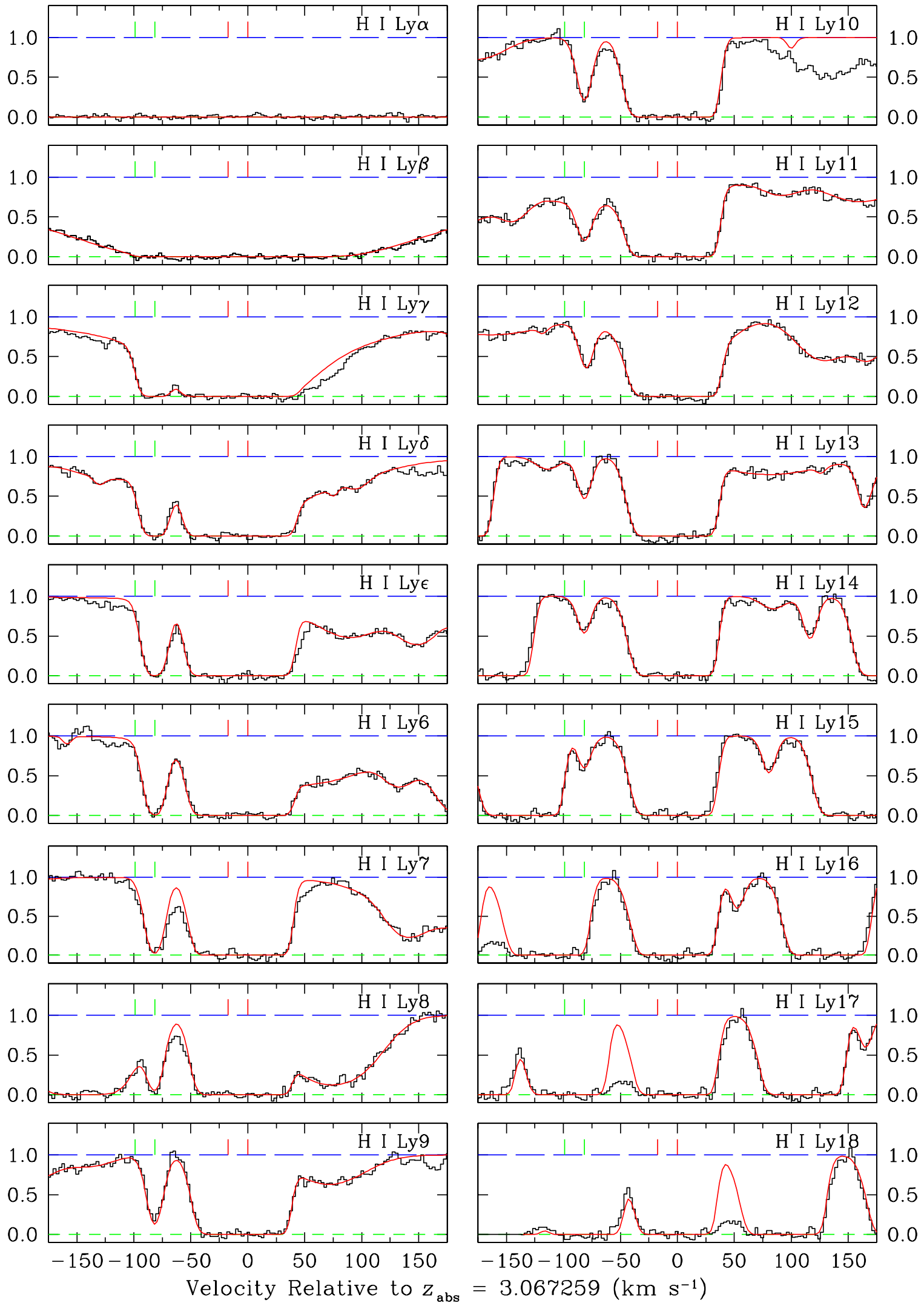

Figure 3. Montage of the full Lyman series absorption in the DLA at $z_{\text {abs }}=3.067259$ toward J1358+6522. The black histogram shows the data, fully adjusted to the best-fitting continuum and zero levels, while the red continuous line is the model fit. The minimum $\chi^{2} /$ dof for this fit is $6282.3 / 6401$. Tick marks above the spectrum indicate the location of the velocity components (red ticks for H I, green ticks for D I).

(A color version of this figure is available in the online journal.) 

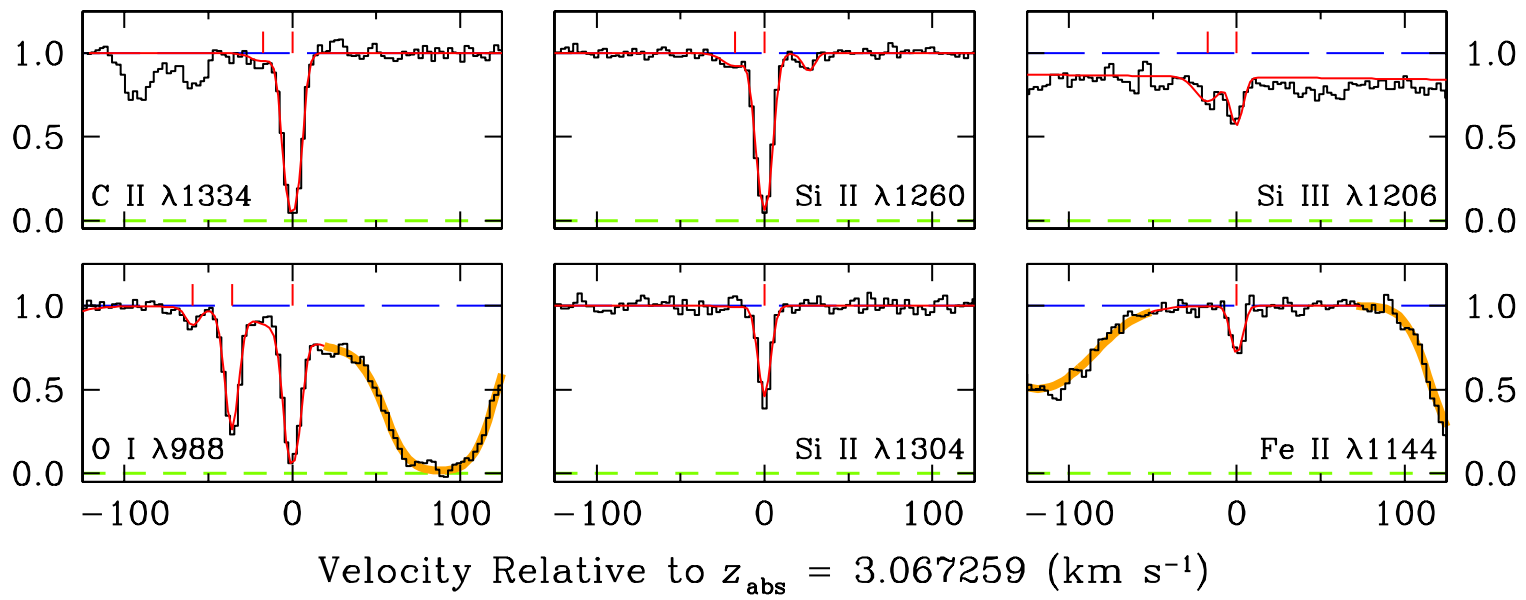

Figure 4. Selection of metal absorption lines for the DLA at $z_{\mathrm{abs}}=3.067259$ toward the QSO J1358+6522 (black histogram, see Cooke et al. 2012). The best-fitting model is shown as the solid red line, while fitted blends are shown as the thick orange lines. In the top panels, the red tick marks above the spectrum indicate the location of the two absorption components, while the bottom panels only display the tick mark for the main absorption component (i.e., component 1a+1b). The long-dashed blue and dashed green lines show the continuum and zero level, respectively. The absorption feature near $+25 \mathrm{~km} \mathrm{~s}^{-1}$ in the Si II $\lambda 1260$ panel is Fe II $\lambda 1260.5$.

(A color version of this figure is available in the online journal.)

Table 1

Best-fitting Model Parameters for the DLA at $z_{\mathrm{abs}}=3.067259$ toward the QSO J1358+6522

\begin{tabular}{|c|c|c|c|c|c|c|c|}
\hline Comp. & $z_{\mathrm{abs}}$ & $\begin{array}{c}T \\
(\mathrm{~K})\end{array}$ & $\begin{array}{c}b_{\text {turb }} \\
\left(\mathrm{km} \mathrm{s}^{-1}\right)\end{array}$ & $\begin{array}{c}\log N(\mathrm{HI}) \\
\left(\mathrm{cm}^{-2}\right)\end{array}$ & $\log (\mathrm{DI} / \mathrm{HI})$ & $\begin{array}{c}\log N(\mathrm{C} \text { II }) \\
\left(\mathrm{cm}^{-2}\right)\end{array}$ & $\begin{array}{c}\log N\left(\mathrm{~N}_{\mathrm{I}}\right) \\
\left(\mathrm{cm}^{-2}\right)\end{array}$ \\
\hline \multirow[t]{2}{*}{$1 \mathrm{a}$} & 3.0672594 & 5700 & 2.4 & 20.21 & $-4.587^{\mathrm{a}}$ & 14.32 & 12.86 \\
\hline & \pm 0.0000005 & \pm 1000 & \pm 0.2 & \pm 0.06 & \pm 0.012 & \pm 0.06 & \pm 0.06 \\
\hline \multirow[t]{2}{*}{$1 b$} & 3.0672591 & 4000 & 8.8 & 20.16 & $-4.587^{\mathrm{a}}$ & $\ldots \mathrm{b}$ &.$^{b}$ \\
\hline & \pm 0.0000018 & \pm 500 & \pm 0.5 & \pm 0.07 & \pm 0.012 & & \\
\hline \multirow[t]{2}{*}{2} & 3.06702 & 13,100 & 8.5 & 17.4 & $-4.587^{\mathrm{a}}$ & 12.30 & $\ldots{ }^{b}$ \\
\hline & \pm 0.00001 & \pm 1500 & \pm 1.4 & \pm 0.03 & \pm 0.012 & \pm 0.15 & \\
\hline
\end{tabular}

\begin{tabular}{|c|c|c|c|c|c|c|}
\hline Comp. & $\begin{array}{c}\log N(\mathrm{O} \mathrm{I}) \\
\left(\mathrm{cm}^{-2}\right)\end{array}$ & $\begin{array}{c}\log N(\mathrm{Al} \text { II }) \\
\left(\mathrm{cm}^{-2}\right)\end{array}$ & $\begin{array}{c}\log N(\mathrm{Si} \mathrm{II}) \\
\left(\mathrm{cm}^{-2}\right)\end{array}$ & $\begin{array}{c}\log N(\mathrm{Si} \text { III }) \\
\quad\left(\mathrm{cm}^{-2}\right)\end{array}$ & $\begin{array}{c}\log N\left(\mathrm{~S}_{\mathrm{II}}\right) \\
\left(\mathrm{cm}^{-2}\right)\end{array}$ & $\begin{array}{c}\log N(\mathrm{Fe} \text { II }) \\
\left(\mathrm{cm}^{-2}\right)\end{array}$ \\
\hline \multirow[t]{2}{*}{$1 \mathrm{a}$} & 14.85 & 11.93 & 13.44 & 11.87 & 13.00 & 13.09 \\
\hline & \pm 0.02 & \pm 0.03 & \pm 0.02 & \pm 0.04 & \pm 0.08 & \pm 0.02 \\
\hline $1 \mathrm{~b}$ & $\ldots{ }^{b}$ & $\ldots{ }^{b}$ & $\ldots{ }^{b}$ & $\ldots{ }^{b}$ & $\ldots$ & $\ldots^{\mathrm{b}}$ \\
\hline \multirow[t]{2}{*}{2} & $\mathrm{~b}^{\mathrm{b}}$ &.$^{b}$ & 11.53 & 11.80 &.$^{b}$ & $\mathrm{~b}^{\mathrm{b}}$ \\
\hline & & & \pm 0.06 & \pm 0.06 & & \\
\hline
\end{tabular}

Notes.

${ }^{\text {a }}$ Forced to be the same for all components.

${ }^{\mathrm{b}}$ Absorption is undetected for this ion in this component.

low-turbulence cloud (component 1a); component $1 \mathrm{~b}$ has less than $1 / 100$ of the metallicity of component $1 \mathrm{a}$. We propose three possible interpretations for this unusual finding: (1) there exists an essentially pristine cloud of gas near the very metal-poor DLA; (2) the metals see a different potential to D I and H I (i.e., the metals have not been entirely mixed into the $\mathrm{HI}$ ); (3) the assumption of a constant kinetic temperature for all elements through the entire sightline is incorrect.

While each of these explanations is interesting in its own right, we stress here that the choice of the cloud model does not affect the derived value of $D / H$ in this system, for the reasons discussed in Section 3.2. Provided that there exists a series of optically thin D I lines, and $\mathrm{H}_{\mathrm{I}} \mathrm{Ly} \alpha$ exhibits damping wings (as indeed is the case for the DLA toward J1358+6522-see Figures 2 and 3), the derived total D I and H I column densities will be independent of the cloud model. We therefore only require that the model provides a good fit to the data.

The final, best-fitting value of the deuterium abundance for the DLA toward J1358+6522 is (expressed as a logarithmic and linear quantity)

$$
\begin{aligned}
\log \left(\mathrm{D}_{\mathrm{I}} / \mathrm{H}_{\mathrm{I}}\right) & =-4.588 \pm 0.012, \\
10^{5} \mathrm{D} \mathrm{I} / \mathrm{HI}_{\mathrm{I}} & =2.58 \pm 0.07,
\end{aligned}
$$

where the quoted error term includes the random (observational) error in addition to the systematic uncertainties that were marginalized over during the fitting process.

\section{THE PRIMORDIAL ABUNDANCE OF DEUTERIUM FROM THE PRECISION SAMPLE}

Including the new $\mathrm{D} / \mathrm{H}$ case reported herein, there are just five metal-poor absorption-line systems in which a precise measure of the primordial abundance of deuterium can be obtained, based on the criteria outlined in Section 2.2. Relevant details of these systems are collected in Table 2. The four DLAs from the literature were all reanalyzed in an identical manner to that described above (Section 3) for J1358+6522. In particular, we 

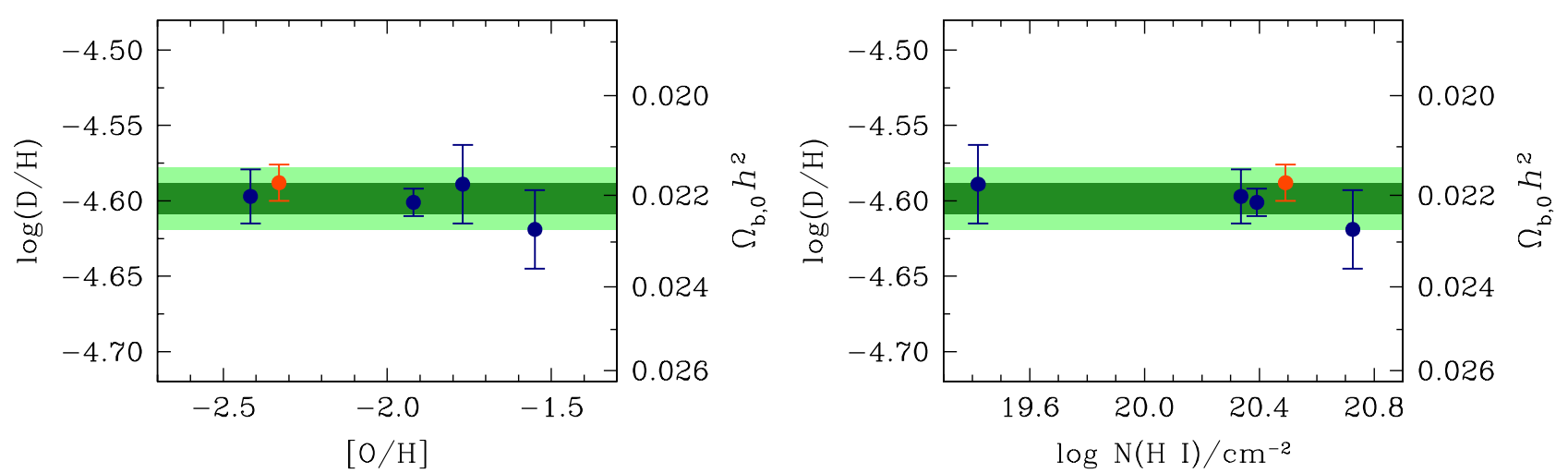

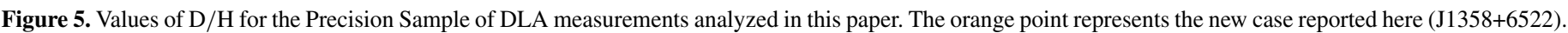

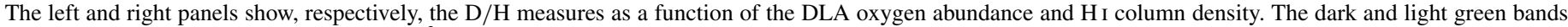

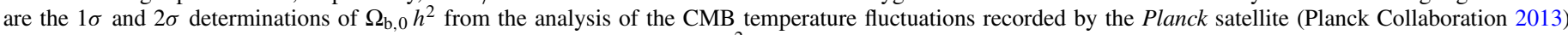
assuming the standard model of physics. The conversion from $\mathrm{D} / \mathrm{H}$ to $\Omega_{\mathrm{b}, 0} h^{2}$ is given by Equations (5) and (6).

(A color version of this figure is available in the online journal.)

Table 2

The Precision Sample of D/H Measurements in QSO Absorption Line Systems

\begin{tabular}{|c|c|c|c|c|c|c|c|c|}
\hline \multirow[b]{2}{*}{ QSO } & \multirow[b]{2}{*}{$z_{\mathrm{em}}$} & \multirow[b]{2}{*}{$z_{\mathrm{abs}}$} & \multirow[b]{2}{*}[\mathrm{O}/\mathrm{H}]{$^{\mathrm{a}}$} & \multicolumn{2}{|c|}{ Literature } & \multicolumn{2}{|c|}{ This Work } & \multirow[b]{2}{*}{ Ref. $^{b}$} \\
\hline & & & & $\begin{array}{c}\log N(\mathrm{HI}) \\
\left(\mathrm{cm}^{-2}\right)\end{array}$ & $\log (\mathrm{D} / \mathrm{H})$ & $\begin{array}{c}\log N(\mathrm{HI}) \\
\left(\mathrm{cm}^{-2}\right)\end{array}$ & $\log (\mathrm{D} / \mathrm{H})$ & \\
\hline HS $0105+1619$ & 2.652 & 2.53651 & -1.77 & $19.42 \pm 0.01$ & $-4.60 \pm 0.04$ & $19.426 \pm 0.006$ & $-4.589 \pm 0.026$ & 1,2 \\
\hline Q0913+072 & 2.785 & 2.61829 & -2.40 & $20.34 \pm 0.04$ & $-4.56 \pm 0.04$ & $20.312 \pm 0.008$ & $-4.597 \pm 0.018$ & $1,3,4$ \\
\hline SDSS J1358+6522 & 3.173 & 3.06726 & -2.33 & $\ldots$ & $\ldots$ & $20.495 \pm 0.008$ & $-4.588 \pm 0.012$ & 1 \\
\hline SDSS J1419+0829 & 3.030 & 3.04973 & -1.92 & $20.391 \pm 0.008$ & $-4.596 \pm 0.009$ & $20.392 \pm 0.003$ & $-4.601 \pm 0.009$ & $1,5,6$ \\
\hline SDSS J1558-0031 & 2.823 & 2.70242 & -1.55 & $20.67 \pm 0.05$ & $-4.48 \pm 0.06$ & $20.75 \pm 0.03$ & $-4.619 \pm 0.026$ & 1,7 \\
\hline
\end{tabular}

Notes.

${ }^{\text {a }}$ We adopt the solar value $\log (\mathrm{O} / \mathrm{H})_{\odot}+12=8.69$ (Asplund et al. 2009).

b References: (1) This work; (2) O'Meara et al. 2001; (3) Pettini et al. 2008b; (4) Pettini et al. 2008a; (5) Pettini \& Cooke 2012; (6) Cooke et al. 2011;

(7) O’Meara et al. 2006.

adopted the same blind analysis strategy and marginalized over the important systematic uncertainties. We refer to this sample of five high-quality measurements as the Precision Sample.

In Table 2, we provide a measure of the total H I column density, along with the associated error. Many of our systems contain more than one component in $\mathrm{H} \mathrm{I}$, and the column density estimates for these multiple components are correlated with one another. To calculate the error on the total Hi column density, we have drawn 10,000 realizations of the component column densities from the covariance matrix. We then calculated the total column density for each realization; in Table 2, we provide the mean and $1 \sigma$ error derived from this Monte Carlo analysis.

We consider the five measures of DI/HI in these DLAs as five independent determinations of the primordial abundance of deuterium, $(\mathrm{D} / \mathrm{H})_{\mathrm{p}}$, for the following reasons: (1) We are not aware of any physical mechanism that would alter the ionization balance of D compared to $\mathrm{H}$. Thus, to our knowledge, D I/ $\mathrm{H} \mathrm{I} \equiv \mathrm{D} / \mathrm{H}$. (2) The degree of astration of D (i.e., its destruction when gas is turned into stars) is expected to be negligible at the low metallicities $([\mathrm{O} / \mathrm{H}]<-1.5)$ of the DLAs considered here (e.g., see Figure 2 of Romano et al. 2006); thus, $(\mathrm{D} / \mathrm{H})_{\mathrm{DLA}}=$ $(\mathrm{D} / \mathrm{H})_{\mathrm{p}}$. (3) The lack of dust in metal-poor DLAs makes it extremely unlikely that selective depletion of D onto grains occurs in the cases considered here (it has been proposed that such a mechanism may be responsible for the local variations in $(\mathrm{D} / \mathrm{H})_{\mathrm{ISM}}$ - see Linsky et al. 2006). (4) The five DLAs sample entirely independent sites in the distant universe.
As can be seen from Table 2 and Figure 5, the five measures of $\mathrm{D} / \mathrm{H}$ in the Precision Sample are in very good mutual agreement, and the dispersion of the measurements is consistent with the errors estimated with our improved analysis. A $\chi^{2}$ test indeed confirms that the five measurements are consistent within $2 \sigma$ of being drawn from a single value of $\mathrm{D} / \mathrm{H}$. We can therefore combine the five independent determinations of $(\mathrm{D} / \mathrm{H})_{\mathrm{DLA}}$ to deduce the weighted mean value of the primordial abundance of deuterium:

$$
\begin{aligned}
\log (\mathrm{D} / \mathrm{H})_{\mathrm{p}} & =-4.597 \pm 0.006 \\
10^{5}(\mathrm{D} / \mathrm{H})_{\mathrm{p}} & =2.53 \pm 0.04 .
\end{aligned}
$$

This value of $(\mathrm{D} / \mathrm{H})_{\mathrm{p}}$ is not markedly different from other recent estimates (Pettini et al. 2008a; Fumagalli et al. 2011; Pettini \& Cooke 2012), but its precision is significantly better than achieved in earlier papers that considered a more heterogeneous set of $(\mathrm{D} / \mathrm{H})_{\mathrm{DLA}}$ determinations. For completeness, we have recalculated the weighted mean for all the known $\mathrm{D} / \mathrm{H}$ measurements listed in Table 2 of Pettini \& Cooke (2012), after updating the $\mathrm{D} / \mathrm{H}$ values of the systems we have reanalyzed here. The resulting weighted mean value of the primordial deuterium abundance is $(\mathrm{D} / \mathrm{H})_{\mathrm{p}}=-4.596 \pm 0.006$. This compares very well with the value derived from the Precision Sample (Equations (3) and (4)). Perhaps this is not surprising, since the literature systems that did not meet our selection criteria (see Section 2.2.1) have larger uncertainties, and thus their contribution to the weighted mean value of $\mathrm{D} / \mathrm{H}$ is relatively low. 


\subsection{The Cosmic Density of Baryons}

Using the most up-to-date calculations of the network of nuclear reactions involved in $\mathrm{BBN}$, the primordial abundance of deuterium is related to the cosmic density of baryons (in units of the critical density), $\Omega_{\mathrm{b}, 0}$, via the following relations (Steigman 2012; G. Steigman 2013, private communication):

$$
\begin{aligned}
(\mathrm{D} / \mathrm{H})_{\mathrm{p}} & =2.55 \times 10^{-5}\left(6 / \eta_{\mathrm{D}}\right)^{1.6} \times(1 \pm 0.03), \\
\eta_{\mathrm{D}} & =\eta_{10}-6(S-1)+5 \xi / 4,
\end{aligned}
$$

where $\eta_{10}=273.9 \Omega_{\mathrm{b}, 0} h^{2}, S=\left[1+7\left(N_{\mathrm{eff}}-3.046\right) / 43\right]^{1 / 2}$ is the expansion factor, and $\xi$ is the neutrino degeneracy parameter (related to the lepton asymmetry by Equation (14) from Steigman 2012). The rightmost term in Equation (5) represents the current $3 \%$ uncertainty in the conversion of $(\mathrm{D} / \mathrm{H})_{\mathrm{p}}$ to $\eta_{\mathrm{D}}$ due to the uncertainties in the relevant nuclear reaction rates (see Section 4.2). For the standard model, $N_{\text {eff }} \simeq 3.046$ and $\xi=0$. In this case, the Precision Sample of $\mathrm{D} / \mathrm{H}$ measurements implies a cosmic density of baryons:

$$
\begin{aligned}
100 \Omega_{\mathrm{b}, 0} h^{2}(\mathrm{BBN})= & 2.202 \pm 0.020(\text { random }) \\
& \pm 0.041 \text { (systematic) }
\end{aligned}
$$

where we have decoupled the error terms from our measurement (i.e., the random error term) and the systematic uncertainty in converting the $\mathrm{D}$ abundance into the baryon density parameter.

As can be seen from Figure 5, this value of $\Omega_{\mathrm{b}, 0} h^{2}$ is in excellent agreement with that derived from the analysis of the CMB temperature fluctuations measured by the Planck satellite (Planck Collaboration 2013):

$$
100 \Omega_{\mathrm{b}, 0} h^{2}(\mathrm{CMB})=2.205 \pm 0.028 .
$$

\subsection{The Current Limitation}

In the era of high-precision cosmology, we feel that it is important to highlight the main limitations affecting the use of $(\mathrm{D} / \mathrm{H})_{\mathrm{p}}$ in the estimation of cosmological parameters. As can be seen from Equation (7), the main source of error is in the conversion of $(\mathrm{D} / \mathrm{H})_{\mathrm{p}}$ to the baryon density parameter $\left(\eta_{\mathrm{D}}\right.$, and hence $\Omega_{\mathrm{b}, 0} h^{2}$ ). In large part, this systematic uncertainty is due to the relative paucity of experimental measures for several nuclear cross sections that are important in the network of BBN reactions, particularly deuteron-deuteron reactions and the $d(p, \gamma)^{3} \mathrm{He}$ reaction rate at the relevant energies (Fiorentini et al. 1998; Nollett \& Burles 2000; Cyburt 2004; Serpico et al. 2004). Since these studies, estimates for the deuteron-deuteron reaction cross sections (Leonard et al. 2006) have improved and their contribution to the error budget has been reduced. The main lingering concern involves the reaction rate $d(p, \gamma)^{3} \mathrm{He}$, for which only a single reliable data set of the $S$-factor is currently available in the relevant energy range (Ma et al. 1997). ${ }^{16}$ The concern for $d(p, \gamma)^{3} \mathrm{He}$ is made worse by the fact that the theoretical and experimental values of the $S$-factor do not agree. This paucity of data, in addition to the difficulties of obtaining an accurate and precise measure of $d(p, \gamma)^{3} \mathrm{He}$ at BBN energies, led Nollett \& Holder (2011) to adopt a theoretical curve for the $S$-factor between 50 and $500 \mathrm{keV}$. This has resulted in the improved conversion given in Equation (5).

\footnotetext{
16 To avoid possible confusion caused by the unfortunate nomenclature, we point out that the $S$-factor in question is directly related to the reaction cross section as a function of energy, $\sigma(E)$. It has nothing to do with the expansion factor $S$ in Equation (6).
}

It is worth pointing out here that a further reduction by a modest factor of two in the uncertainty of the conversion from $(\mathrm{D} / \mathrm{H})_{\mathrm{p}}$ to $\eta_{\mathrm{D}}$ would make the precision of $\Omega_{\mathrm{b}, 0} h^{2}(\mathrm{BBN})$ from the DLA Precision Sample comparable to that of $\Omega_{\mathrm{b}, 0} h^{2}(\mathrm{CMB})$ achieved by the Planck mission (see Equations (7) and (8)).

\section{BEYOND THE STANDARD MODEL}

In this section we combine the results presented here with those of the CMB analysis by the Planck Collaboration to place bounds on some parameters denoting new physics beyond the standard model of cosmology and particle physics. In particular, we use the Markov-Chain Monte Carlo chains from the combined analysis of the Planck temperature fluctuations (Planck Collaboration 2013), the low-multipole WMAP polarization data ( $l<23$; Bennett et al. 2013), and high-resolution CMB data (see Planck Collaboration 2013 for an appropriate list of references). Throughout, we refer to this combined data set as Planck+WP+highL, for consistency with the work by the Planck Collaboration (2013). In what follows, we have assumed that $N_{\text {eff }}$ and the baryon-to-photon ratio remained unchanged from the epoch of BBN to the epoch of recombination.

\subsection{Combined CMB+BBN Measure of $N_{\text {eff }}$ and $\Omega_{b, 0} h^{2}$}

It has long been known that the mass fraction of ${ }^{4} \mathrm{He}, Y_{\mathrm{P}}$, is potentially a very sensitive probe of additional light degrees of freedom (Steigman et al. 1977). Unfortunately, systematic uncertainties in the determination of $Y_{\mathrm{P}}$ have limited its use as an effective probe of $N_{\text {eff }}$ (see Figure 8 of Steigman 2012). However, Nollett \& Holder (2011) (see also Cyburt 2004) have recently highlighted the potential of using precise measurements of the primordial deuterium abundance in conjunction with observations of the CMB to place a strong, independent bound on $N_{\text {eff }}$.

In the left panel of Figure 6 , we show the current $1 \sigma$ and $2 \sigma$ confidence contours for $N_{\text {eff }}$ and $\Omega_{\mathrm{b}, 0} h^{2}$ derived from the Planck+WP+highL CMB analysis ${ }^{17}$ (green) and from the BBNderived $(\mathrm{D} / \mathrm{H})_{\mathrm{p}}$ reported here (blue). The combined confidence bounds are overlaid as red contours. In what follows, it is instructive to recall that the CMB-only bounds are (Planck Collaboration 2013)

$$
\begin{array}{r}
100 \Omega_{\mathrm{b}, 0} h^{2}=2.23 \pm 0.04, \\
N_{\mathrm{eff}}=3.36 \pm 0.34 .
\end{array}
$$

(Note that solving simultaneously for $\Omega_{\mathrm{b}, 0} h^{2}$ and $N_{\text {eff }}$ leads to a slightly different best-fitting value of $\Omega_{\mathrm{b}, 0} h^{2}$ than that obtained for the standard model; see Equations (8) and (9)). For comparison, from the joint $\mathrm{BBN}+\mathrm{CMB}$ analysis we deduce

$$
\begin{array}{r}
100 \Omega_{\mathrm{b}, 0} h^{2}=2.23 \pm 0.04, \\
N_{\mathrm{eff}}=3.28 \pm 0.28 .
\end{array}
$$

Thus, combining $(\mathrm{D} / \mathrm{H})_{\mathrm{p}}$ with the $\mathrm{CMB}$ does not significantly change the uncertainty in $\Omega_{\mathrm{b}, 0} h^{2}$, but does reduce the error on $N_{\text {eff }}$ by $\sim 20 \%$. The uncertainty on $N_{\text {eff }}$ could be reduced further by an improvement in the cross section of the $d(p, \gamma)^{3} \mathrm{He}$ (see right panel of Figure 6, and Section 4.2). Based on the current bound on $N_{\text {eff }}$ from $\mathrm{CMB}+(\mathrm{D} / \mathrm{H})_{\mathrm{p}}$, we can nevertheless rule out the existence of an additional (sterile) neutrino (i.e.,

\footnotetext{
17 We used the base cosmology set with $N_{\text {eff }}$ added as a free parameter (see Section 6.4.4 of Planck Collaboration 2013).
} 

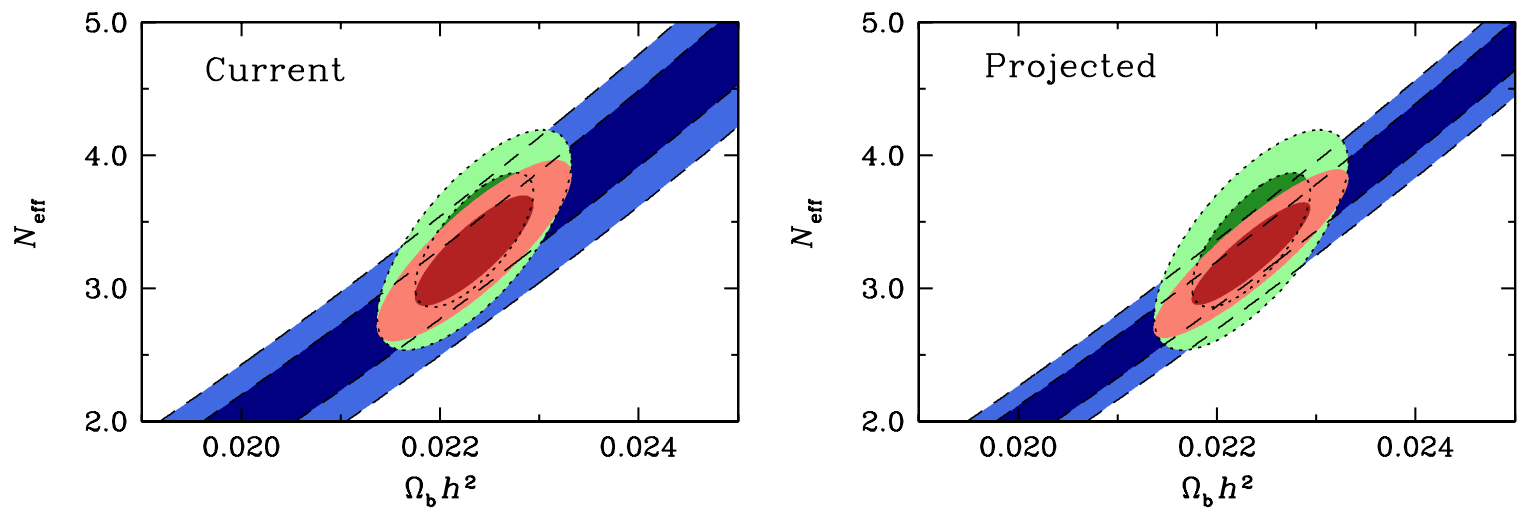

Figure 6. $1 \sigma$ and $2 \sigma$ confidence contours (dark and light shades, respectively) for $N_{\mathrm{eff}}$ and $\Omega_{\mathrm{b}, 0} h^{2}$ derived from the primordial deuterium abundance (blue), the CMB (green), and the combined confidence contours (red). The left panel illustrates the current situation, while the right panel shows the effect of reducing the uncertainty in the conversion from $(\mathrm{D} / \mathrm{H})_{\mathrm{p}}$ to $\Omega_{\mathrm{b}, 0} h^{2}$ by a factor of two (see discussion in Section 4.2). Dashed and dotted lines indicate the hidden contour lines for BBN and CMB bounds, respectively.

(A color version of this figure is available in the online journal.)

$\left.N_{\text {eff }}=4.046\right)$ at $99.3 \%$ confidence (i.e., $\sim 2.7 \sigma$ ), provided that $N_{\text {eff }}$ and $\eta_{10}$ remained unchanged between BBN and recombination. However, as noted recently by Steigman (2013), if the CMB photons are heated after the neutrinos have decoupled [for example, by a weakly interacting massive particle that annihilates to photons], $N_{\text {eff }}$ will be less than 3.046 for three standard model neutrinos; a sterile neutrino can in principle exist even when $N_{\text {eff }}<4.046$.

Looking to the future, $Y_{\mathrm{P}}$ has contours that are almost orthogonal to those of the CMB and $(\mathrm{D} / \mathrm{H})_{\mathrm{p}}$ (see, e.g., Steigman 2007). Thus, measures of $Y_{\mathrm{P}}$ that are not limited by systematic uncertainties could potentially provide a very strong bound, when combined with $(\mathrm{D} / \mathrm{H})_{\mathrm{p}}$, on the number of equivalent neutrinos during the epoch of $\mathrm{BBN}$, independently of $\mathrm{CMB}$ observations.

Following improvements in the He I emissivity calculations (Porter et al. 2012, 2013), there have been two recent reassessments of $Y_{\mathrm{P}}$, by Izotov et al. (2013) and by Aver et al. (2013), respectively. Izotov et al. (2013) find $Y_{\mathrm{P}}=0.253 \pm 0.003$; this value includes a small correction of -0.001 to reflect the fact that their Cloudy modeling overpredicts $Y_{\mathrm{P}}$ by this amount at the lowest metallicities-see their Figure 7(a). Aver et al. (2013) find $Y_{\mathrm{P}}=0.254 \pm 0.004$ from the average of all the low-metallicity $\mathrm{H}$ II regions in their sample. Thus, the values deduced by these two teams are in good mutual agreement; in the analysis that follows we adopt $Y_{\mathrm{P}}=0.253 \pm 0.003$ from Izotov et al. (2013). Using the following conversion relation for $Y_{\mathrm{P}}$ (Steigman 2012; G. Steigman 2013, private communication):

$$
\begin{aligned}
Y_{\mathrm{P}} & =0.2469 \pm 0.0006+0.0016\left(\eta_{\mathrm{He}}-6\right), \\
\eta_{\mathrm{He}} & =\eta_{10}+100(S-1)-575 \xi / 4,
\end{aligned}
$$

combined with the Izotov et al. (2013) measure of $Y_{\mathrm{P}}$, we derive the following BBN-only bound on the baryon density and the effective number of neutrino species:

$$
\begin{array}{r}
100 \Omega_{\mathrm{b}, 0} h^{2}=2.28 \pm 0.05, \\
N_{\text {eff }}=3.50 \pm 0.20 .
\end{array}
$$

The corresponding contours are shown in Figure 7. Thus, it appears that even with the most recent reappraisals of the primordial abundance of ${ }^{4} \mathrm{He}$ by Izotov et al. (2013) and Aver et al. (2013), there is better agreement (within the standard model) between $(\mathrm{D} / \mathrm{H})_{\mathrm{p}}$ and the CMB than between $(\mathrm{D} / \mathrm{H})_{\mathrm{p}}$ and $Y_{\mathrm{P}}$.

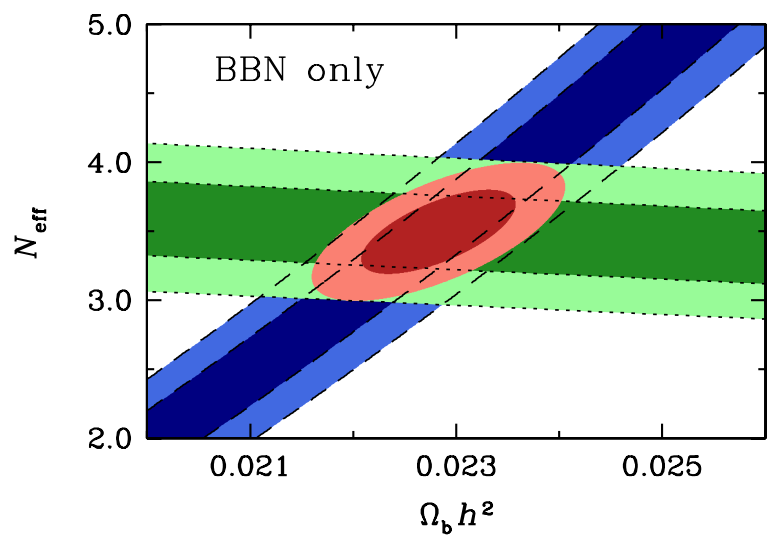

Figure 7. $1 \sigma$ and $2 \sigma$ confidence contours (dark and light shades, respectively) for $N_{\text {eff }}$ and $\Omega_{\mathrm{b}, 0} h^{2}$ derived from the primordial deuterium abundance (blue), the primordial He mass fraction (green), and the combined confidence contours (red). Dashed and dotted lines indicate the hidden contour lines for $(D / H)_{p}$ and $Y_{\mathrm{P}}$ bounds, respectively.

(A color version of this figure is available in the online journal.)

\subsection{Deuterium and the Lepton Asymmetry}

In the past, the primordial deuterium abundance has been commonly used as a tool for measuring the present-day universal density of baryons (see, e.g., Steigman 2007), and more recently as a probe of the effective number of neutrino families (Cyburt 2004; Nollett \& Holder 2011; Pettini \& Cooke 2012; see also Section 5.1). Here, we demonstrate that precise measures of the primordial deuterium abundance (in combination with the CMB) can also be used to estimate the neutrino degeneracy parameter, $\xi$, which is related to the lepton asymmetry by Equation (14) from Steigman (2012).

Steigman (2012) recently suggested that combined estimates for $(\mathrm{D} / \mathrm{H})_{\mathrm{p}}, Y_{\mathrm{P}}$, and a measure of $N_{\text {eff }}$ from the CMB can provide interesting limits on the neutrino degeneracy parameter $(\xi \leqslant 0.079,2 \sigma$; see also Serpico \& Raffelt 2005; Popa \& Vasile 2008; Simha \& Steigman 2008). By combining $(\mathrm{D} / \mathrm{H})_{\mathrm{p}}$ and $Y_{\mathrm{P}}$, this approach effectively removes the dependence on $\Omega_{\mathrm{b}, 0} h^{2}$. Using the conversion relations for $(\mathrm{D} / \mathrm{H})_{\mathrm{p}}$ and $Y_{\mathrm{P}}$ (Equations (5)-(6) and 13-14) and the current best determination of $Y_{\mathrm{P}}(0.253 \pm 0.003$; Izotov et al. 2013), in addition to the Planck+WP+highL ${ }^{18}$ constraint on $N_{\text {eff }}$ and the precise 18 We used the base cosmology set with $N_{\text {eff }}$ and $Y_{\mathrm{P}}$ added as free parameters
(see Section 6.4.5 of Planck Collaboration 2013). 
determination of $(\mathrm{D} / \mathrm{H})_{\mathrm{p}}$ reported here, we derive a $2 \sigma$ upper limit on the neutrino degeneracy parameter, $|\xi| \leqslant 0.064$, based on the approach by Steigman (2012).

We propose that an equally powerful technique for estimating $\xi$ does not involve removing the dependence on $\Omega_{\mathrm{b}, 0} h^{2}$ by combining $(\mathrm{D} / \mathrm{H})_{\mathrm{p}}$ and $Y_{\mathrm{P}}$, as in Steigman (2012). Instead, one can obtain a measure of both $\Omega_{\mathrm{b}, 0} h^{2}$ and $N_{\text {eff }}$ from the CMB and use either $(\mathrm{D} / \mathrm{H})_{\mathrm{p}}$ or $Y_{\mathrm{P}}$ to obtain two separate measures of $\xi$. This has the clear advantage of decoupling $(\mathrm{D} / \mathrm{H})_{\mathrm{p}}$ and $Y_{\mathrm{P}}$; any systematic biases in either of these two values could potentially bias the measure of $\xi$. Separating $(\mathrm{D} / \mathrm{H})_{\mathrm{p}}$ and $Y_{\mathrm{P}}$ also allows one to check that the two estimates agree with one another.

Our calculation involved a Monte Carlo technique, whereby we generated random values from the Gaussian-distributed primordial $\mathrm{D} / \mathrm{H}$ abundance measurements, while simultaneously drawing random values from the (correlated) distribution between $\Omega_{\mathrm{b}, 0} h^{2}$ and $N_{\mathrm{eff}}$ from the Planck+WP+highL CMB data (Planck Collaboration 2013). ${ }^{19}$ Using Equation (19) from Steigman (2012, equivalent to Equation (6) here), we find $\xi_{\mathrm{D}}=+0.05 \pm 0.13$ for $(\mathrm{D} / \mathrm{H})_{\mathrm{p}}$, leading to a $2 \sigma$ upper limit of $\left|\xi_{\mathrm{D}}\right| \leqslant 0.31$.

With the technique outlined above, we have also computed the neutrino degeneracy parameter from the current observational bound on $Y_{\mathrm{P}}$. For this calculation, we have used the MCMC chains from the Planck+WP+highL CMB base cosmology with $N_{\text {eff }}$ and $Y_{\mathrm{P}}$ added as free parameters. In this case, the CMB distribution was weighted by the observational bound on $Y_{\mathrm{P}}$ $\left(Y_{\mathrm{P}}=0.253 \pm 0.003\right.$; Izotov et al. 2013). Using Equations (19)-(20) from Steigman (2012, equivalent to Equations (6) and (14) here), we find $\xi_{\mathrm{D}}=+0.04 \pm 0.15$ for $(\mathrm{D} / \mathrm{H})_{\mathrm{p}}$ and $\xi_{\mathrm{He}}=$ $-0.010 \pm 0.027$ for $Y_{\mathrm{P}}$. These values translate into corresponding $2 \sigma$ upper limits $\left|\xi_{\mathrm{D}}\right| \leqslant 0.34$ and $\left|\xi_{\mathrm{He}}\right| \leqslant 0.064$. Combining these two constraints then gives $\xi=-0.008 \pm 0.027$, or $|\xi| \leqslant 0.062(2 \sigma)$.

Alternatively, if we assume that the effective number of neutrino species is consistent with three standard model neutrinos (i.e., $N_{\text {eff }} \simeq 3.046$ ), we obtain the following BBN-only bound on the neutrino degeneracy parameter by combining $(\mathrm{D} / \mathrm{H})_{\mathrm{p}}$ and $Y_{\mathrm{P}}, \xi=-0.026 \pm 0.015$, or $|\xi| \leqslant 0.056(2 \sigma)$. We therefore conclude that all current estimates of the neutrino degeneracy parameter, and hence the lepton asymmetry, are consistent with the standard model value, $\xi=0$.

From the above calculations, it is clear that $Y_{\mathrm{P}}$ is the more sensitive probe of any lepton asymmetry, as is already appreciated. However, $(\mathrm{D} / \mathrm{H})_{\mathrm{p}}$ offers an additional bound on $\xi$ that is complementary to that obtained from $Y_{\mathrm{P}}$. We note further that, if the uncertainty in the conversion of $(\mathrm{D} / \mathrm{H})_{\mathrm{p}}$ to $\eta_{\mathrm{D}}$ could be reduced by a factor of two, the bound on $\xi_{\mathrm{D}}$ would be reduced by $15 \%$, corresponding to a $1 \sigma$ uncertainty on $\xi$ of $\sim 0.11$ from the $\mathrm{CMB}$ and deuterium measurements alone.

\section{SUMMARY AND CONCLUSIONS}

We have reported a new precise measurement of the primordial abundance of deuterium in a very metal-poor damped Ly $\alpha$ system at $z_{\mathrm{abs}}=3.06726$ toward the QSO SDSS J1358+6522. Furthermore, we have reanalyzed self-consistently all literature systems that meet a set of strict criteria (four cases). These criteria were imposed to identify the systems where both accurate

\footnotetext{
19 Rather than drawing values of $\Omega_{\mathrm{b}, 0} h^{2}$ and $N_{\text {eff }}$ from the appropriate distribution, we instead used the Markov-Chain Monte Carlo chains provided by the Planck science team, which are available at

http://www.sciops.esa.int/wikiSI/planckpla/index.php?title=

Cosmological_Parameters\&instance=Planck_Public_PLA
}

and precise measures of $\mathrm{D} / \mathrm{H}$ could potentially be obtained. The new system, plus the four from the literature, constitute the Precision Sample of DLAs that are best suited for a precise determination of $(\mathrm{D} / \mathrm{H})_{\mathrm{p}}$. Our reanalysis was performed blind (to remove human bias) and took advantage of a new software package that we have specifically developed for the measurement of D/H in QSO absorption line systems. From the analysis of this sample, we draw the following conclusions.

1. The very metal-poor DLA $([\mathrm{Fe} / \mathrm{H}]=-2.88 \pm 0.03)$ toward SDSS J1358+6522 provides a strong bound on the primordial abundance of deuterium, $(\mathrm{D} / \mathrm{H})_{\mathrm{p}}=(2.58 \pm 0.07) \times$ $10^{-5}$. A weighted mean of the five systems in the Precision Sample gives the most stringent limit on $(\mathrm{D} / \mathrm{H})_{\mathrm{p}}$ to date: $(\mathrm{D} / \mathrm{H})_{\mathrm{p}}=(2.53 \pm 0.04) \times 10^{-5}$. The corresponding baryon density is $100 \Omega_{\mathrm{b}, 0} h^{2}=2.202 \pm 0.046$, assuming the standard model of particle physics with three families of neutrinos and no lepton asymmetry. This value is in excellent agreement with $100 \Omega_{\mathrm{b}, 0} h^{2}(\mathrm{CMB})=2.205 \pm 0.028$ deduced from the analysis of Planck observations of the CMB.

2. The main limitation in using $(\mathrm{D} / \mathrm{H})_{\mathrm{p}}$ for cosmological parameter estimation is the conversion of $(D / H)_{p}$ into the ratio of baryons to photons. In particular, modern measurements of the cross section for the reaction $d(p, \gamma)^{3} \mathrm{He}$ at energies between 50 and $500 \mathrm{keV}$, where there currently exists a paucity of reliable data, are highly desirable. We estimate that a factor of two improvement in the conversion from $(\mathrm{D} / \mathrm{H})_{\mathrm{p}}$ to $\Omega_{\mathrm{b}, 0} h^{2}$ would provide a measurement of the universal baryon density from $\mathrm{D} / \mathrm{H}$ as precise as that derived from the published Planck data.

3. By combining our $\mathrm{D} / \mathrm{H}$ determination with Planck observations of the $\mathrm{CMB}$, we can place a tight bound on both $\Omega_{\mathrm{b}, 0} h^{2}$ and $N_{\text {eff }}$. The best-fitting parameters so derived are $100 \Omega_{\mathrm{b}, 0} h^{2}=2.23 \pm 0.04$ and $N_{\text {eff }}=3.28 \pm 0.28$. We therefore rule out the existence of an additional (sterile) neutrino at $99.3 \%$ confidence, provided that $N_{\text {eff }}$ and $\eta_{10}$ remained unchanged from $\mathrm{BBN}$ to recombination. The uncertainty on $N_{\text {eff }}$ could be reduced further by a more accurate set of reaction cross sections for $d(p, \gamma)^{3} \mathrm{He}$ and, to a lesser degree, $d+d$.

4. For the first time, we have combined the Planck CMB results with our measure of $(\mathrm{D} / \mathrm{H})_{\mathrm{p}}$ to place a bound on the neutrino degeneracy parameter, $\xi_{\mathrm{D}}=+0.05 \pm 0.13$. When including the current best estimate of the ${ }^{4} \mathrm{He}$ mass fraction, $Y_{\mathrm{P}}$, derived from metal-poor $\mathrm{H}_{\mathrm{II}}$ regions, the combined bound on the neutrino degeneracy parameter is $\xi=-0.008 \pm 0.027$, or $|\xi|<0.062$ at $2 \sigma$.

We conclude by re-emphasizing that the most metal-poor DLAs are potentially the best environments where both accurate and precise measures of the primordial abundance of deuterium can be obtained. A combined effort to measure anew several important nuclear reaction cross sections, in addition to dedicated searches for the rare metal-poor DLAs that exhibit resolved deuterium absorption, are the next steps necessary to pin down further the value of $(\mathrm{D} / \mathrm{H})_{\mathrm{p}}$. This approach offers a promising and exciting avenue to test for departures from the standard model of cosmology and particle physics.

We are grateful to the staff astronomers at the ESO VLT and Keck Observatory for their assistance with the observations. We are indebted to Gary Steigman for kindly communicating ahead of publication the latest fitting formulae used in this work, and 


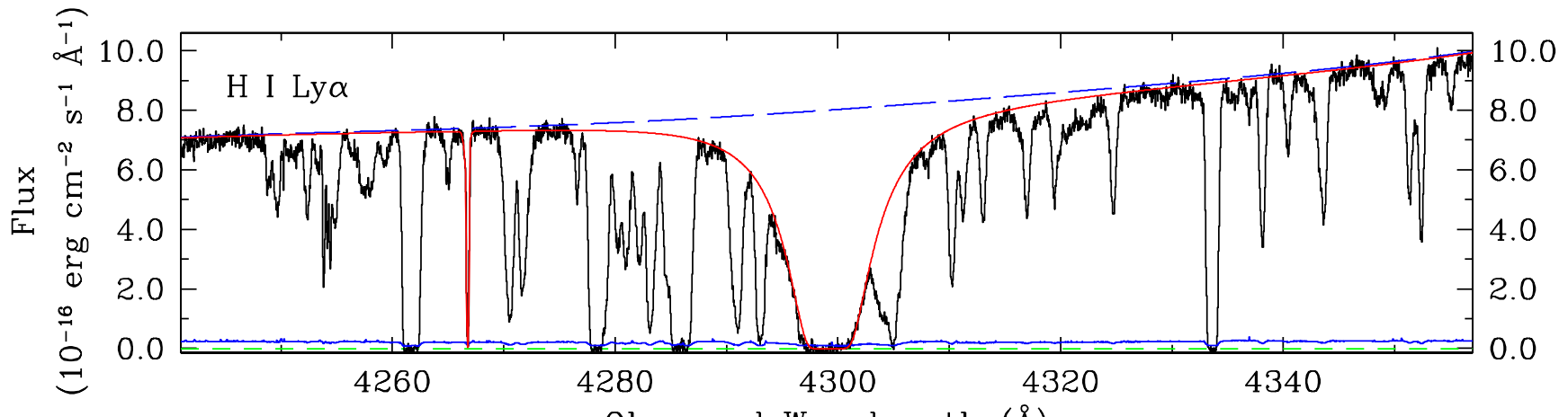

Observed Wavelength $(\AA)$
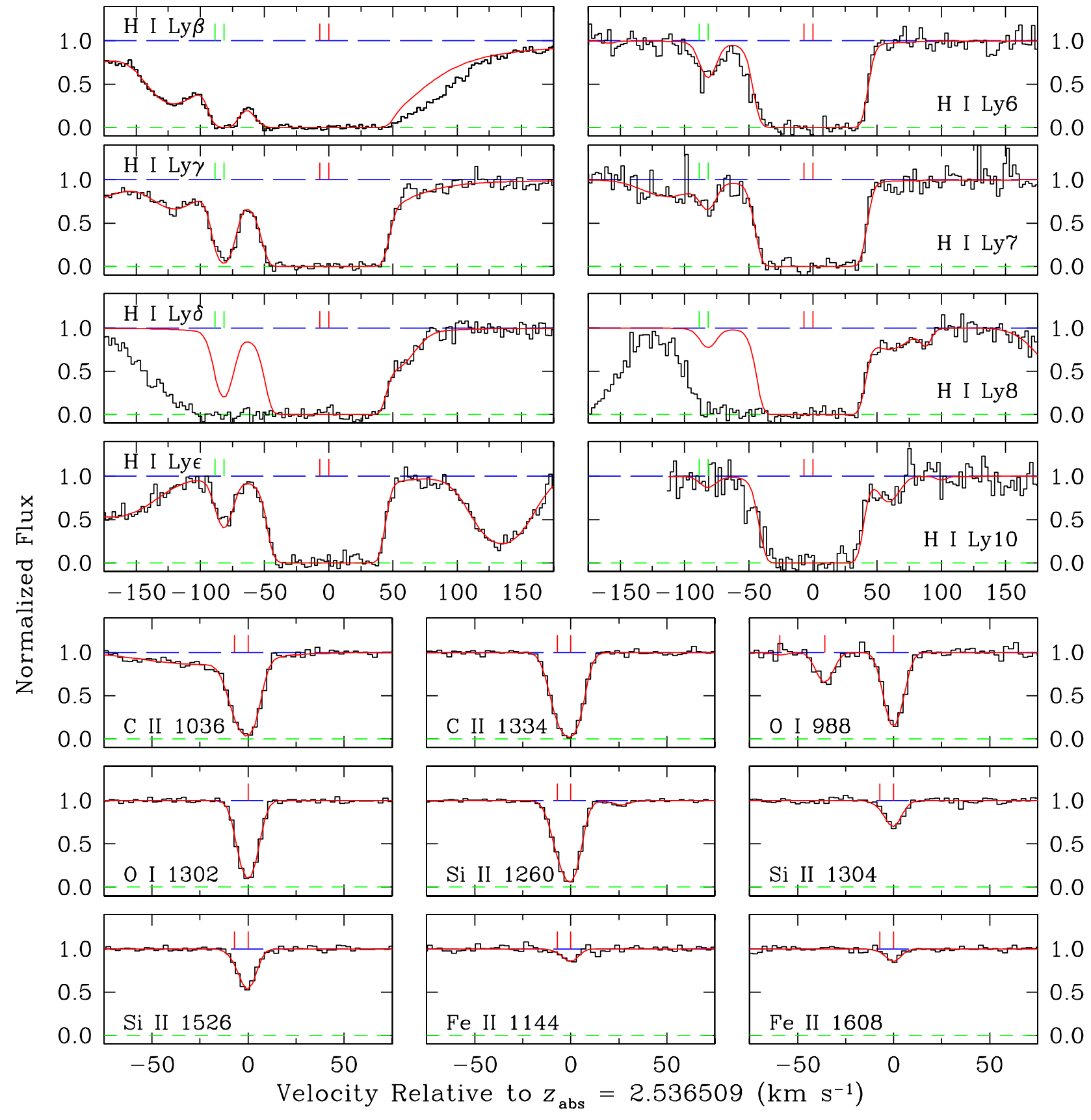

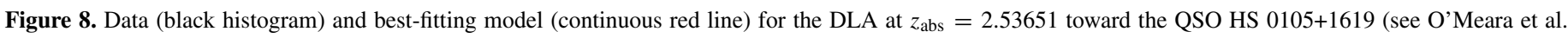

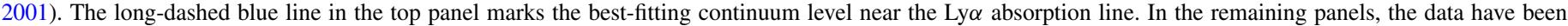

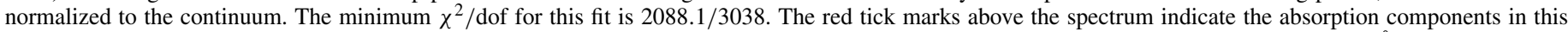

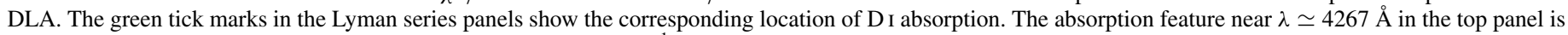
Si III $\lambda 1206.5$ at the redshift of the DLA. The feature near $+25 \mathrm{~km} \mathrm{~s}^{-1}$ in the Si II $\lambda 1260$ panel is Fe II $\lambda 1260.5$.

(A color version of this figure is available in the online journal.) 

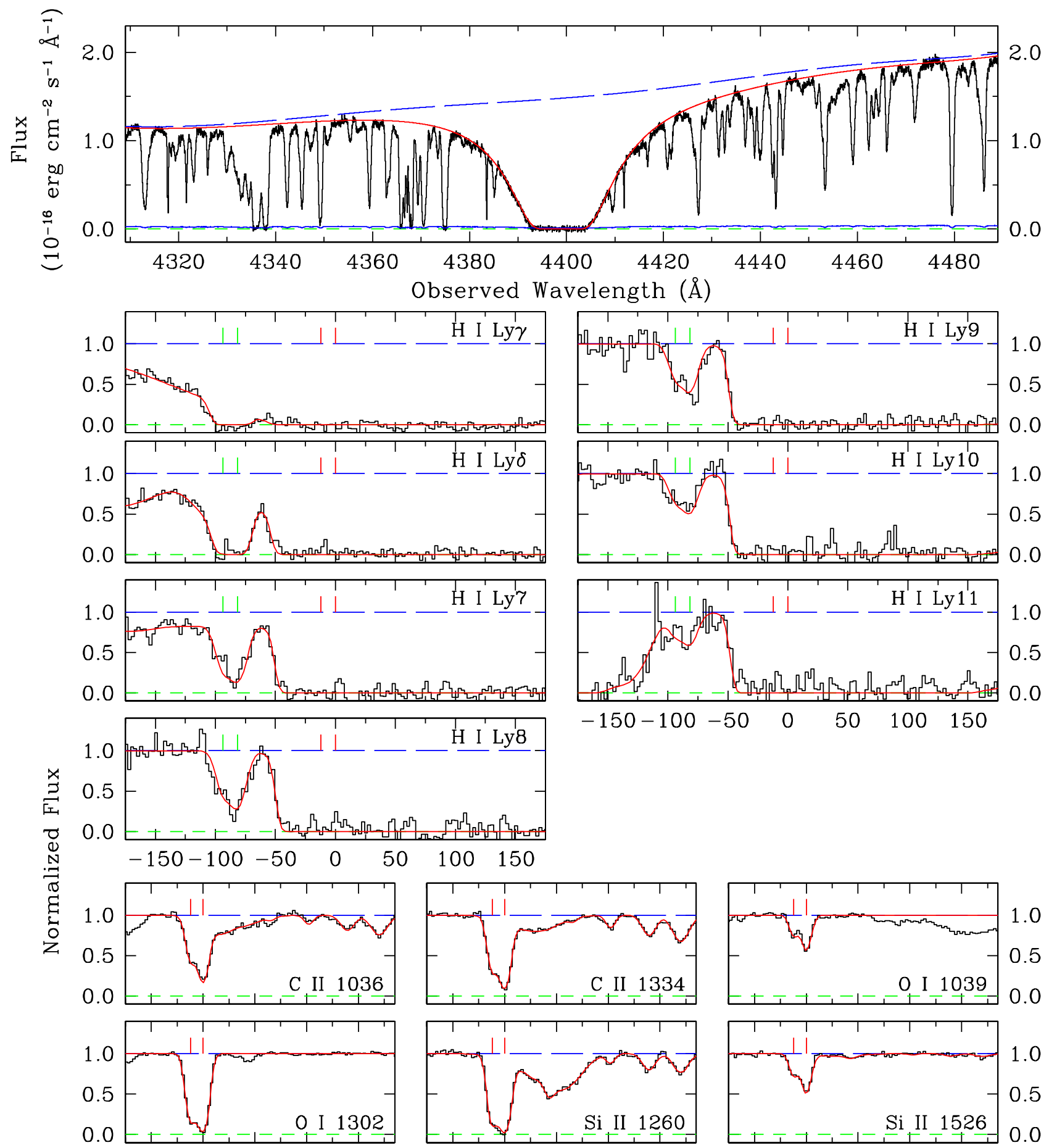

H I Ly?
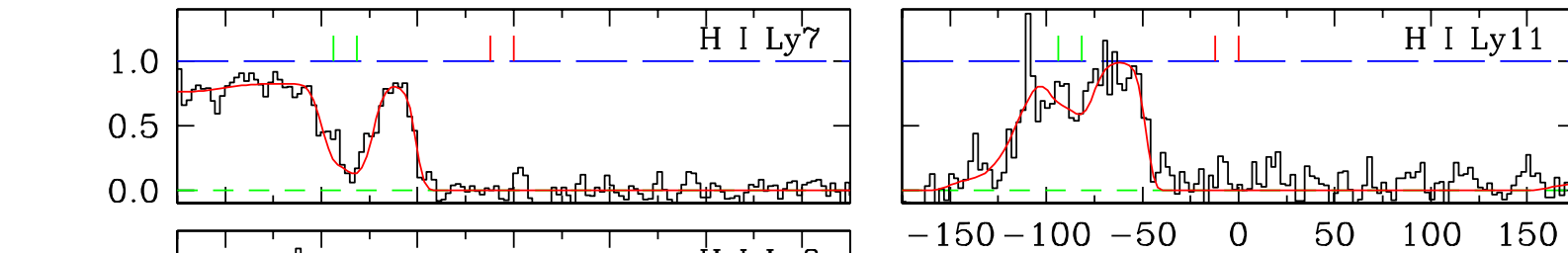

$$
\text { 产 }
$$
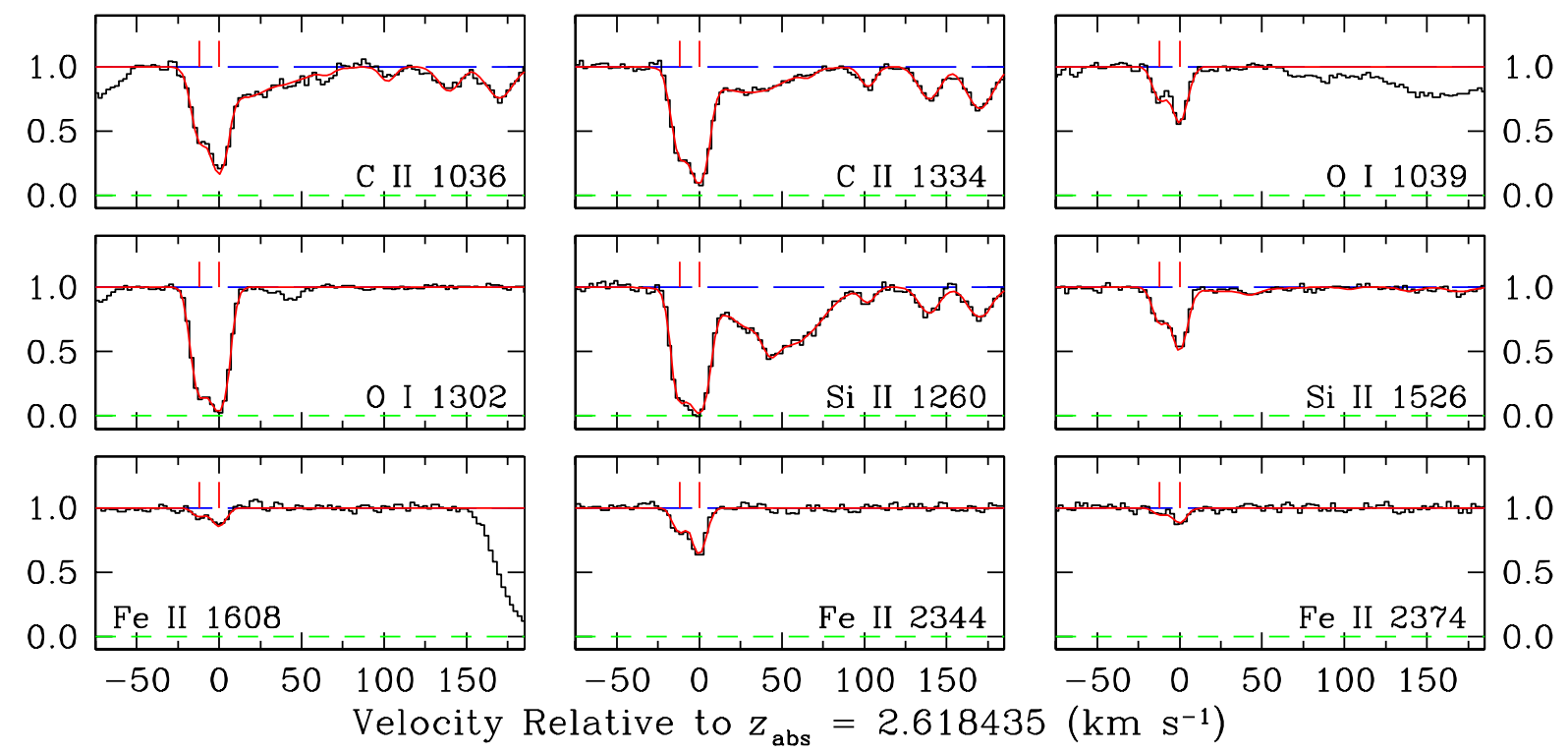

Figure 9. Same as Figure 8 for the DLA at $z_{\mathrm{abs}}=2.61829$ toward the QSO Q0913+072 (see Pettini et al. 2008a). The minimum $\chi^{2} /$ dof for this fit is $5539.8 / 4473$. (A color version of this figure is available in the online journal.) 

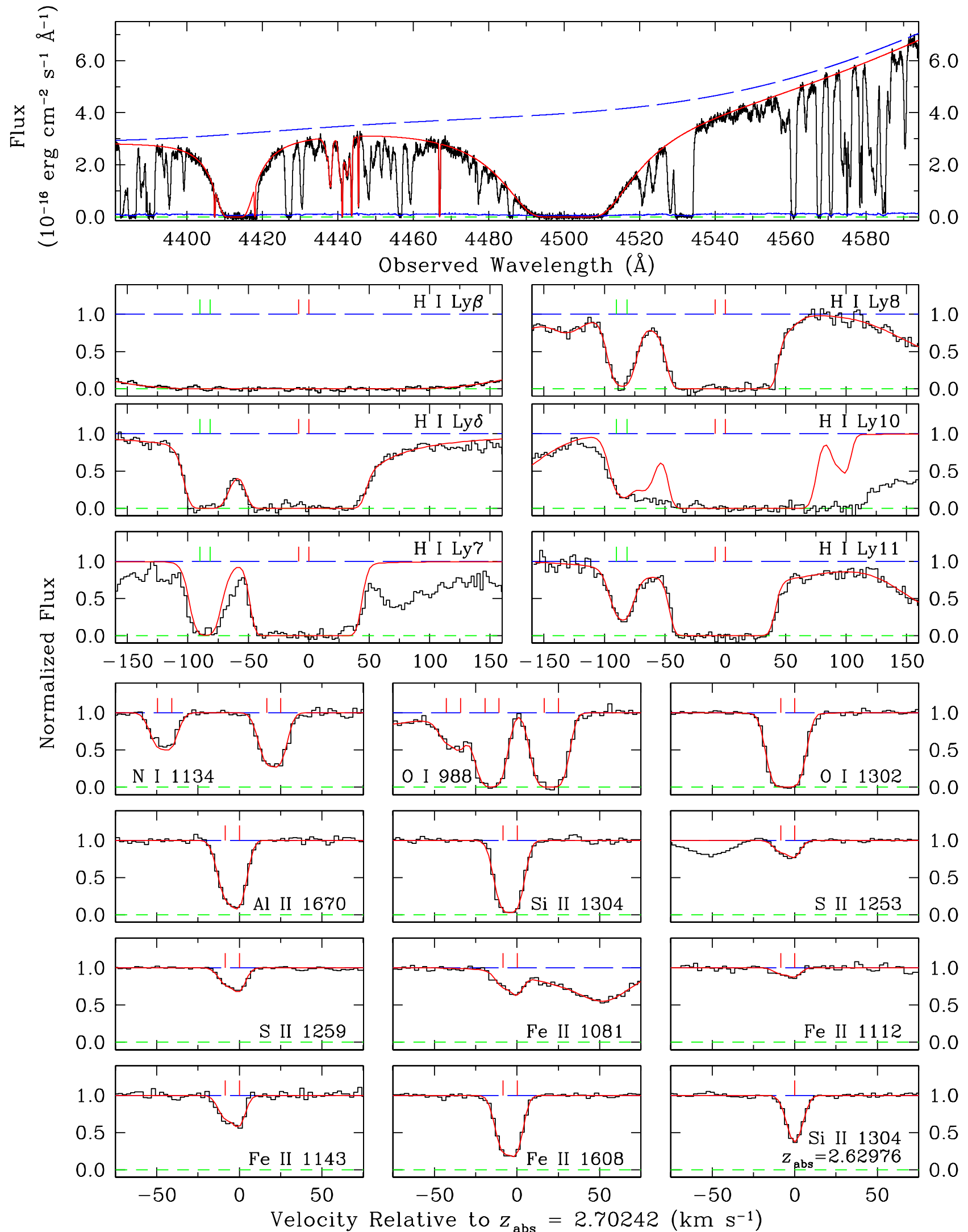

Figure 10. Same as Figure 8 for the DLA at $z_{\text {abs }}=2.70242$ toward the QSO J1558-0031 (see O'Meara et al. 2006). The DLA near $4500 \AA \AA$ is the system where D/H can be measured. The lower redshift system (at $\left.z_{\mathrm{abs}}=2.62976\right)$ is included in the simultaneous fit to the higher redshift system (see the bottom-right panel for an example Si II line profile in the low-redshift system). The minimum $\chi^{2} /$ dof for this fit is $4754.4 / 4700$. In the top panel, several absorption features from metal lines at $z_{\text {abs }}=2.70242$ (Si II $\lambda \lambda 1190.4,1193.3$ near $4407 \AA$ and $4418 \AA$, respectively, N I $\lambda \lambda 1200$ and including some additional blends near $4440 \AA$, and Si III $\lambda 1206.5$ near $4467 \AA$ ) are indicated in red. The "extra" absorption in the trough of H I Ly10 is due to H I Ly $\epsilon$ from the system at redshift $z_{\text {abs }}=2.62976$. The absorption feature at $+100 \mathrm{~km} \mathrm{~s}^{-1}$ in this same panel corresponds to O I $\lambda 919.6$ at $z_{\mathrm{abs}}=2.70242$.

(A color version of this figure is available in the online journal.) 
for providing valuable comments on the manuscript. We also thank an anonymous referee who provided useful suggestions that improved the presentation of this work. Discussions, advice, and help with various aspects of the work described in this paper were provided by Antony Lewis, Jordi Miralda-Escudé, Paolo Molaro, Ken Nollett, Pasquier Noterdaeme, John O’Meara, Jason X. Prochaska, Signe Riemer-Sørenson, Donatella Romano, and John Webb. We thank the Hawaiian people for the opportunity to observe from Mauna Kea; without their hospitality, this work would not have been possible. R.J.C. is partially supported by NSF grant AST-1109447. R.A.J. is supported by an NSF Astronomy and Astrophysics Postdoctoral Fellowship under award AST-1102683. M.T.M. thanks the Australian Research Council for a QEII Research Fellowship (DP0877998) and a Discovery Project grant (DP130100568).

\section{APPENDIX}

\section{MODEL FITS TO LITERATURE SYSTEMS}

In this Appendix Figures 8-10 show portions of the spectra of the metal-poor DLAs from the literature that were reanalyzed in the present work. Further details are given in Section 2.2.

\section{REFERENCES}

Adams, T. F. 1976, A\&A, 50, 461

Asplund, M., Grevesse, N., Sauval, A. J., \& Scott, P. 2009, ARA\&A, 47, 481

Aver, E., Olive, K. A., Porter, R. L., \& Skillman, E. D. 2013, JCAP, 11, 017

Bennett, C. L., Larson, D., Weiland, J. L., et al. 2013, ApJS, 208, 20

Burles, S., \& Tytler, D. 1998a, ApJ, 499, 699

Burles, S., \& Tytler, D. 1998b, ApJ, 507, 732

Cooke, R., Pettini, M., \& Murphy, M. T. 2012, MNRAS, 425, 347

Cooke, R., Pettini, M., Steidel, C. C., Rudie, G. C., \& Nissen, P. E. 2011, MNRAS, 417, 1534

Cyburt, R. H. 2004, PhRvD, 70, 023505

Erni, P., Richter, P., Ledoux, C., \& Petitjean, P. 2006, A\&A, 451, 19 Fiorentini, G., Lisi, E., Sarkar, S., \& Villante, F. L. 1998, PhRvD, 58, 063506

Fumagalli, M., O’Meara, J. M., \& Prochaska, J. X. 2011, Sci, 334, 1245

Izotov, Y. I., Stasinska, G., \& Guseva, N. G. 2013, A\&A, 558, A57
Lampton, M., Margon, B., \& Bowyer, S. 1976, ApJ, 208, 177

Lee, H.-W. 2013, ApJ, 772, 123

Leonard, D. S., Karwowski, H. J., Brune, C. R., et al. 2006, PhRvC, 73, 045801

Linsky, J. L., Draine, B. T., Moos, H. W., et al. 2006, ApJ, 647, 1106

Ma, L., Karwowski, H. J., Brune, C. R., et al. 1997, PhRvC, 55, 588

Markwardt, C. B. 2009, in ASP Conf. Ser. 411, Astronomical Data Analysis Software and Systems XVIII, ed. D. A. Bohlender, D. Durand, \& P. Dowler (San Francisco, CA: ASP), 251

Meléndez, J., Casagrande, L., Ramírez, I., Asplund, M., \& Schuster, W. J. 2010, A\&A, 515, L3

Morton, D. C. 2003, ApJS, 149, 205

Nollett, K. M., \& Burles, S. 2000, PhRvD, 61, 123505

Nollett, K. M., \& Holder, G. P. 2011, arXiv:1112.2683

Noterdaeme, P., Petitjean, P., Carithers, W. C., et al. 2012, A\&A, 547, L1

O’Meara, J. M., Burles, S., Prochaska, J. X., et al. 2006, ApJL, 649, L61

O’Meara, J. M., Tytler, D., Kirkman, D., et al. 2001, ApJ, 552, 718

Penprase, B. E., Prochaska, J. X., Sargent, W. L. W., Toro-Martinez, I., \& Beeler, D. J. 2010, ApJ, 721, 1

Pettini, M., \& Cooke, R. 2012, MNRAS, 425, 2477

Pettini, M., Zych, B. J., Murphy, M. T., Lewis, A., \& Steidel, C. C. 2008a, MNRAS, 391, 1499

Pettini, M., Zych, B. J., Steidel, C. C., \& Chaffee, F. H. 2008b, MNRAS, 385, 2011

Planck Collaboration 2013, arXiv:1303.5076

Popa, L. A., \& Vasile, A. 2008, JCAP, 06, 028

Porter, R. L., Ferland, G. J., Storey, P. J., \& Detisch, M. J. 2012, MNRAS, 425, L28

Porter, R. L., Ferland, G. J., Storey, P. J., \& Detisch, M. J. 2013, MNRAS, 433, L89

Prochaska, J. X., \& Wolfe, A. M. 2009, ApJ, 696, 1543

Romano, D., Tosi, M., Chiappini, C., \& Matteucci, F. 2006, MNRAS, 369,295

Sbordone, L., Bonifacio, P., Caffau, E., et al. 2010, A\&A, 522, A26

Serpico, P. D., Esposito, S., Iocco, F., et al. 2004, JCAP, 12, 010

Serpico, P. D., \& Raffelt, G. G. 2005, PhRvD, 71, 127301

Simha, V., \& Steigman, G. 2008, JCAP, 08, 011

Spite, F., \& Spite, M. 1982, A\&A, 115, 357

Steigman, G. 2001, in Proc. STScI Symp. Ser. 15, The Dark Universe: Matter, Energy and Gravity, ed. M. Livio (Baltimore, MD: Cambridge Univ. Press), 46

Steigman, G. 2007, ARNPS, 57, 463

Steigman, G. 2012, arXiv:1208.0032

Steigman, G. 2013, PhRvD, 87, 103517

Steigman, G., Schramm, D. N., \& Gunn, J. E. 1977, PhLB, 66, 202

Vogt, S. S., Allen, S. L., Bigelow, B. C., et al. 1994, Proc. SPIE, 2198, 362

Wagoner, R. V., Fowler, W. A., \& Hoyle, F. 1967, ApJ, 148, 3 